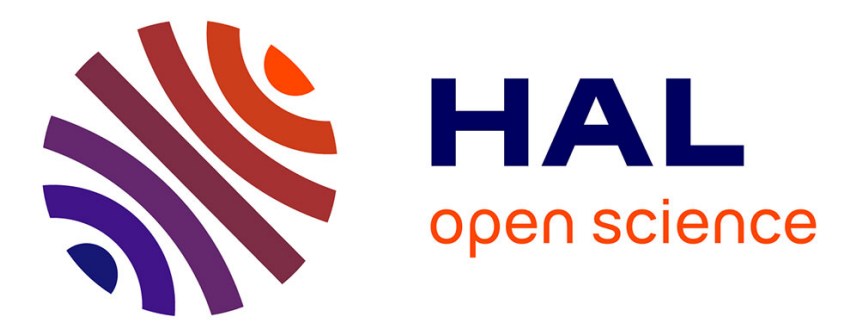

\title{
Cutting-edge mass spectrometry methods for the multi-level structural characterization of antibody-drug conjugates
}

Alain Beck, Guillaume Terral, François Debaene, Elsa Wagner-Rousset, Julien Marcoux, Marie-Claire Janin-Bussat, Olivier Colas, Alain Van Dorsselaer, Sarah Cianférani

\section{To cite this version:}

Alain Beck, Guillaume Terral, François Debaene, Elsa Wagner-Rousset, Julien Marcoux, et al.. Cutting-edge mass spectrometry methods for the multi-level structural characterization of antibody-drug conjugates. Expert Review of Proteomics, 2016, 13 (2), pp.157-183. 10.1586/14789450.2016.1132167 . hal-02335539

\section{HAL Id: hal-02335539 \\ https://hal.science/hal-02335539}

Submitted on 19 Mar 2021

HAL is a multi-disciplinary open access archive for the deposit and dissemination of scientific research documents, whether they are published or not. The documents may come from teaching and research institutions in France or abroad, or from public or private research centers.
L'archive ouverte pluridisciplinaire HAL, est destinée au dépôt et à la diffusion de documents scientifiques de niveau recherche, publiés ou non, émanant des établissements d'enseignement et de recherche français ou étrangers, des laboratoires publics ou privés. 
Publisher: Taylor \& Francis

Journal: Expert Review of Proteomics

DOI: $10.1586 / 14789450.2016 .1132167$

Review

Cutting-edge mass spectrometry methods for the multi-level structural characterization of antibody-drug conjugates

Alain Beck ${ }^{(1) *}$, Guillaume Terral ${ }^{(2,3)}$, François Debaene ${ }^{(2,3) \neq}$, E. Wagner-Rousset ${ }^{(1)}$, Julien $\operatorname{Marcoux}^{(2,3) \Psi}$, Marie-Claire Janin-Bussat ${ }^{(1)}$, Olivier Colas ${ }^{(1)}$, Alain Van Dorsselaer ${ }^{(2,3)}$ and Sarah Cianférani ${ }^{(2,3)} *$

${ }^{(1)}$ Centre d'Immunologie Pierre-Fabre (CIPF), 5 Av. Napoléon III, BP 60497, 74164 SaintJulien-en-Genevois, France

${ }^{(2)}$ BioOrganic Mass Spectrometry Laboratory (LSMBO), IPHC, Université de Strasbourg, 25 rue Becquerel, 67087 Strasbourg, France

${ }^{(3)}$ IPHC, CNRS, UMR7178, 67087 Strasbourg, France

*alain.beck@pierre-fabre.com, *sarah.cianferani@unistra.fr

Present address:

Institute of Pharmacology and Structural Biology - UMR 5089 - 205 Route de Narbonne, 31077 Toulouse, France

${ }^{*}$ Sanofi, Analytics \& Formulation Dpt - 9, quai Jules Guesde, 94403 Vitry-sur-Seine, France 


\section{Abstract}

Antibody drug conjugates (ADCs) are highly cytotoxic drugs covalently attached via conditionally stable linkers to monoclonal antibodies (mAbs) and are among the most promising next-generation empowered biologics for cancer treatment. ADCs are more complex than naked mAbs, as the heterogeneity of the conjugates adds to the inherent microvariability of the biomolecules. The development and optimization of $\mathrm{ADCs}$ rely on improving their analytical and bioanalytical characterization by assessing several critical quality attributes, namely the distribution and position of the drug, the amount of naked antibody, the average drug to antibody ratio, and the residual druglinker and related product proportions. Here brentuximab vedotin (Adcetris®) and trastuzumab emtansine (Kadcyla $\left.{ }^{\circledR}\right)$, the first and gold-standard hinge-cysteine and Tysine drug conjugates, respectively, were chosen to develop new mass spectrometry (MS) methods and to improve multiplelevel structural assessment protocols.

\section{Keywords}

Antibody drug conjugate, bioanalysis, brentuximab vedotin, drug to antibody ratio, IdeS, ion mobility MS, native MS, mass speetrometry, trastuzumab emtansine

\section{Abbreviation List}

MS, mass spectrometry; ESI, electrospray ionization; ADC, antibody-drug-conjugate; DAR, drug-to-antibody ratio; HDX, hydrogen/deuterium exchange; BV, brentuximab vedotin; TDM1, trastuzumab emtansine; HOS, higher order structure; mAb, monoclonal antibody; HER2, human epidermal growth factor receptor 2; human immunoglobilin G, IgG; UHPLC, ultra-high performance liquid chromatography; MS/MS, tandem mass spectrometry; SEC, size exclusion chromatography; HIC, hydrophobic interaction chromatography; SMCC, succinimidyl-4-(N-maleimidomethyl)cyclohexane-1-carboxylate; CQA, critical quality attribute; rpHPLC, reversed-phase high performance liquid chromatography; CE, capillary electrophoresis; CE-SDS, capillary electrophoresis-sodium dodecyl sulphate ; IEF, 
isolelectrofocusing; icIEF, imaged capillary isoelectrofocusing (icIEF); SRM, selected reaction monitoring; Q-TOF, quadrupole time-of-flight; IM-MS, ion mobility mass spectrometry; ACN, acetonitrile; FA, formic acid; DTT, dithiothreitol; TCEP, tris(2carboxyethyl)phosphine; IdeS, immunoglobulin-degrading enzyme from Streptococcus pyogenes; mcMMAF, maleimidocaproyl-monomethyl Auristatin F; vcMMAE, valinecitrulline-monomethyl Auristatin E; XRD, X-ray diffraction; NMR, nuclear magnetic resonance; PK, pharmacokinetics; CD, circular dichroism; DSC, differential scanning calorimetry; UV, ultraviolet; Vis, visible; ELISA, enzyme-like immunosorbent assay. 


\section{Introduction}

With advances in protein engineering technologies, monoclonal antibodies (mAbs) and their derivatives have emerged as the largest drug class in human therapeutics [1]. More than 60 antibody based pharmaceuticals such as ADCs, radio-immunoconjugates, bispecific antibodies, Fab fragments, Fc-fusion proteins and peptides have already been approved to treat cancer, autoimmune diseases, and more recently, to lower cholesterol levels [2]. More than 50 others are under phase III clinical trials [3], with an approval rate $(\neg 20 \%)$ which compares favorably with that of other new chemical entities $(\sim 5 \%)$. The success of mAbs stems from their unique properties namely their high specificity and affinity (in the nM to $\mathrm{pM}$ range), their long circulating half-lives (up to 20 days) and their ability to induce immune cell effector response. For oncology therapy however, first generation mAbs are often inefficient or face resistance. To overcome these limitations, several families of armed antibodies are emerging - including radio-immunoconjugates [4], antibody-drug conjugates (ADCs, another class of immunoconjugates) [5], immunotoxins [6] and immunocytokines [7], among which ADCs have so far proved the most successful, with two drugs already on the market. Brentuximab vedotin (BV, marketed as Adcetris ${ }^{\circledR}$ by Seattle Genetics/Takeda) is indicated for the treatment of hematological malignancies (Hodgkin's lymphoma and systemic anaplastic large-cell lymphoma) while trastuzumab emtansine (T-DM1, marketed as Kadcyla $\AA$ by Genentech/Roche) has been approved for the treatment of breast cancer patients resistant to human epidermal growth factor receptor 2 (HER2) [8,9]. The ADCs in development target a wide range of cancers [10,11]. Many of these new compounds have emerged from a better understanding of structure-function relationships, which have mainly been achieved thanks to state-of-the art mass spectrometry (MS) methods [12,13], but also from lessons learned from pharmaceutical and clinical developments. An example of this process is Mylotarg ${ }^{\circledR}$ (gemtuzumab ozogamicin), which in 2000 became the first ADC 
approved by the FDA but was then withdrawn by the sponsor in 2010. One of the motivations for its withdrawal was its poor chemistry manufacturing and control characteristics, chiefly the presence of $\sim 50 \%$ unconjugated mAbs competing with the ADC for antigen recognition and cell internalization [14-16]. This has been corrected for inotuzumab ozogamicin, another ADC from the same family, currently in clinical phase III, whose production process ensures the removal of naked antibodies.

The present review aims to draw an exhaustive picture of MS-based methodologies available for the characterization of ADCs at different levels, those of the intact conjugated protein, subunits thereof, peptides, and free drug-linker and related products (respectively the top, middle, bottom, and drug-linker levels, respectively). A particular focus will be MS approaches that allow ADCs to be analyzed in near native conditions and provide an assessment of their higher order structures.

\section{ADC architecture}

Antibody-drug conjugates $(\sim 154 \mathrm{kDa})$ consist of a recombinant monoclonal antibody $(\sim 148$ $\mathrm{kDa})$ covalently attached by a synthetic linker to a highly cytotoxic agent $(0.3-1.5 \mathrm{kDa})$ [17]. The three structural components of an ADC are thereby (Figure 1): i) an antibody, which specifically recognizes cancer cells by binding to an overexpressed membrane antigen; ii) a payload, which is a potent warhead that inhibits the growth of the cancer cells upon its release; and iii) a cleavable or non-cleavable linker, which covalently binds the two moieties together. In ADCs therefore, the proven antigen-specific selectivity of mAbs is complemented by the potency of highly cytotoxic molecule [18]. As a result, the systemic toxicity associated with traditional chemotherapeutic treatments is reduced and the therapeutic index improved. 


\section{Antibody carrier}

From a structural standpoint, therapeutic chimeric, humanized, and human G immunoglobilins (IgGs) are tetrameric glycoproteins with molecular weights of approximately $150 \mathrm{kDa}$. They comprise two heavy chains ( $\sim 50 \mathrm{kDa}$ each) and two light chains ( $25 \mathrm{kDa}$ each). Disulfide bridges (16 for IgG1 and IgG4; 18 for IgG2) and noncovalent interactions maintain their three-dimensional (3D) structure $(\mathrm{H} 2 \mathrm{~L} 2$ homoheterodimers). The heavy and light chains are linked by one disulfide bond and the heavy chains by two (for IgG1 and IgG4) or three (for IgG2) disulfide bonds located in a short hinge domain. The other 12 disulfide bridges are intramolecular and delimit six different globular domains. Antigen binding is mediated by the variable domains, mainly by three loops connecting individual $\beta$-strands in each domain named complementarity determining regions. Like natural IgGs, all recombinant antibodies contain an Asn-X-Ser/Thr-X' (where X and $\mathrm{X}^{\prime}$ are any amino acid other than proline) consensus sequence for $\mathrm{N}$-glycosylation in their heavy chain $\mathrm{CH} 2$ constant domain. On average, $\operatorname{IgG}$ glycans account for just $2-3 \%$ of the total mass of the antibody.

\section{Cytotoxic warheads}

The vast majority of ADCs either approved or at the clinical trial stage utilize auristatin and maytansine derivatives - tubulin polymerization inhibitors - as payloads. Nevertheless an increasing number of clinical-stage ADCs exploit calicheamycins (DNA cleaving agents), duocarmycins (DNA alkylating agents), doxorubicins (DNA intercalating agents), pyrrolobenzodiazepines (DNA cross-linking agents), or SN38, an irinotecan metabolite (topoisomerase I inhibitor). These compounds are all highly toxic for cells and are more hydrophobic than the antibodies [17,19]. 


\section{Linker properties}

A plethora of linkers have been designed to connect the drug and antibody moieties. Most are stable in the bloodstream at physiological $\mathrm{pH}(7.4)$ and temperature but are labile once the ADC is inside the cells targeted by the antibody. They may contain conditional cleavage sites (acid-, lysosomal protease- or glutathione-sensitive) or not (depend on degradation of the $\mathrm{mAb}$ component in the lysosomes). In addition, their polarity (the number of charged residues) can be optimized to limit aggregation and increase or reduce the number of bystander cells killed.

a) Brentuximab vedotin

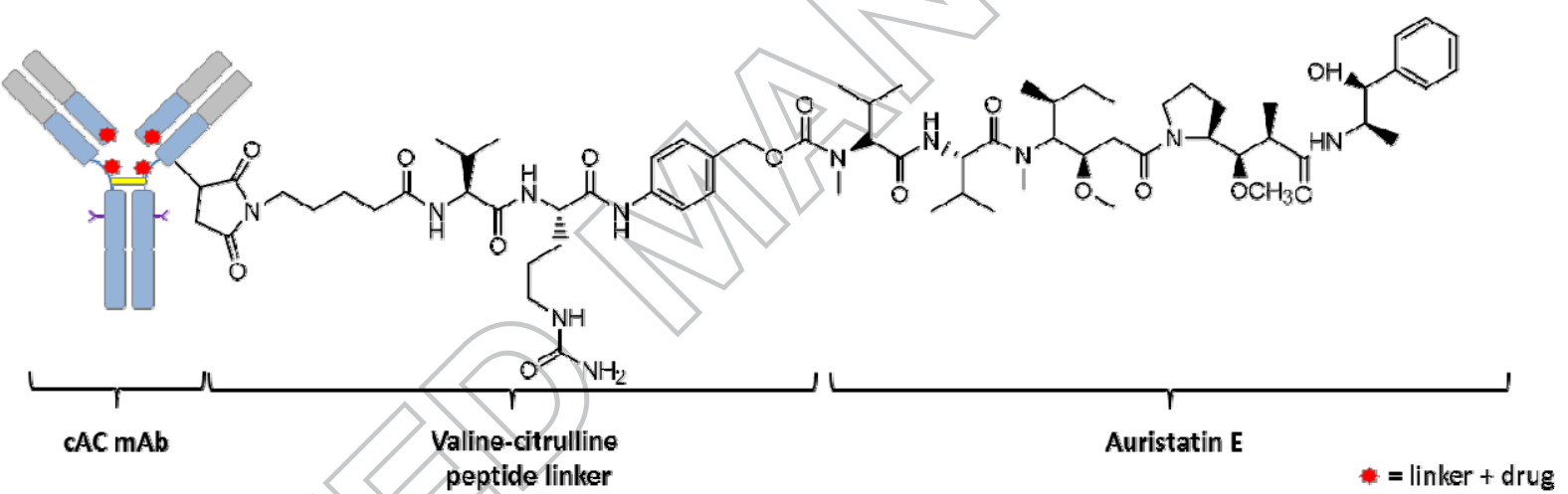

b) Trastuzumab emtansine

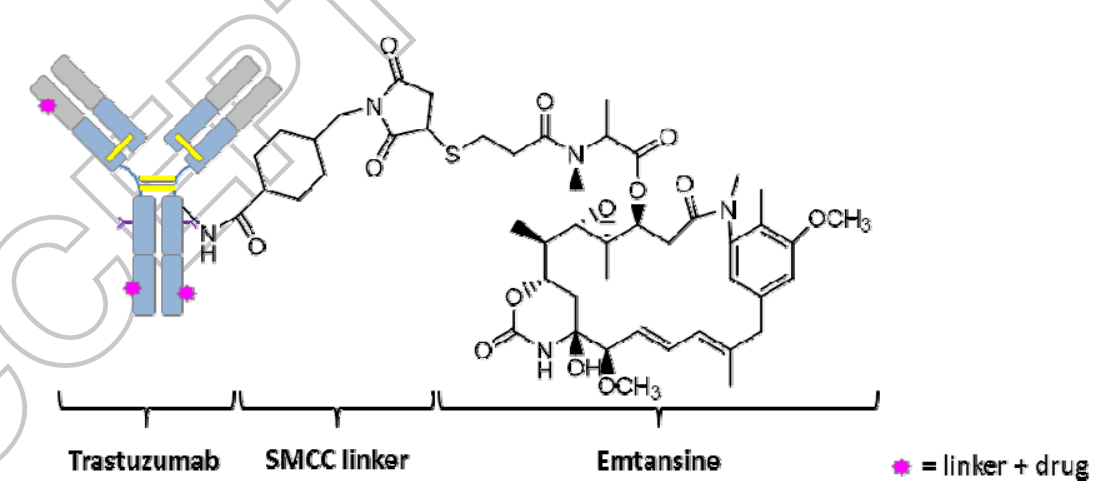

Figure 1. Schematic representation of the structure of the two FDA- and EMA-approved ADCs, which comprise an antibody, a linker and a payload. (a) Brentuximab vedotin (auristatin, protease cleavable linker, Cys-conjugation); (b) trastuzumab emtansine (maytansinoid, non-reducible thioether linkage, Lys-conjugation). 


\section{Drug conjugation}

Drug conjugation is most frequently achieved via reactions on side chains of two different amino acids: lysine side-chain amines or cysteine thiol groups after reduction of the interchain disulfide bonds [20]. The conjugated drugs already on the market-BV, a hinge cysteinelinked ADC, and T-DM1, a surface exposed lysine conjugate - are illustrative of two main ADC families currently in clinical trials (Figure 1).

Cysteine-linked ADCs are typically generated by partially reducing the interchain disulfides of the antibody hinge before alkylation with a cytotoxic agent, most frequently through maleimide chemistry [21,22]. This class of ADCs forms controlled mixtures of molecular species with a variable number of drug molecules per antibody (known as the drugto-antibody ratio, DAR), a different location of the cytotoxic drug for a particular DAR, and a mixture of covalently and non-covalently associated light- and heavy-chain subdomains (Figure 2a).

Lysine conjugates are most commonly assembled via the formation of amide bonds between the epsilon amino group of endogenous lysine residues and activated esters [23,24]. Most IgGs contain -90 lysine residues but only 32 cysteines, among which 8 only are involved in the interchain disulfide bridges of chimeric, humanized and human IgG1. Therefore, although the number of drug molecules incorporated on average per antibody is similar [25], lysine conjugation yields a much more heterogeneous ADC population than cysteine conjugation does. In the reference lysine conjugate T-DM1 for instance, 40 out of the 88 lysine residues were found to be solvent-exposed. Lysine conjugation of T-DM1 proceeds through the reaction of trastuzumab with the heterobifunctional linker succinimidyl-4-(Nmaleimidomethyl)cyclohexane-1-carboxylate (SMCC), producing a linker-modified intermediate. The cytotoxic agent DM1, which contains a free thiol group, is added in a 
second step and reacts with the maleimide to produce the thioether-linked drug conjugate. This results in an average DAR for T-DM1 of 3.5.

The instability of the chemical link between the mAb and the drug in systemic circulation is problematic (eg retro-Michael deconjugation) however. This drug loss modifies the composition of the ADC, potentially diminishing both the amount of drug delivered to the tumor site and its off-target toxicity and overall safety. Other points of concern stem from current bioconjugation methods that yield mixtures of antibodies with different DARs, and within each DAR species, different conjugation sites, each species potentially having different toxicities and ADME properties [26]. Last but not least, the lack of high-resolution analytical techniques for their structural characterization is hampering the development of nextgeneration ADCs.

To extend the therapeutic window and to produce more homogeneous ADCs, a number of strategies have recently been developed allowing more site-specific conjugation [27]. This can be achieved either by adding engineered cysteine residues at specific sites without disruption of the interchain disulfide bonds [28-31] or by adding engineered peptide tags recognized by microbial transglutaminases to specifically transamidate amine-containing drug linkers attached to glutamine residues [32,33]. Alternatively, unnatural amino acids can be inserted into the primary sequence of mAbs to provide a chemical handle on their conjugation [34,35]. As an alternative to maleimide conjugation moreover, the ongoing development of new heterobifunctional reagents should facilitate the production of more stable ADCs [36,37]. To our knowledge, two to four of these next-generation ADCs have already reached the stage of phase I clinical trials $[31,38,39]$ and have shown an improved therapeutic index in pre-clinical toxicology studies [40]. 


\section{The ADC analytical toolbox: a combination of native and denaturing methods}

A large number of orthogonal analytical and bioanalytical methods are needed for the characterization of ADCs [9,41]. Table 1 lists the analytical, structural and functional assays reported in ADC quality control monographs as having been used either for batch release or for characterization and structural assessment $[13,20,42,43]$.

\section{ADCs present an increased level of complexity compared to naked mAbs.}

As discussed above, most of the current clinical-stage ADCs consist of a heterogeneous population of species with a variable number of drug molecules per@antibody (Figure 2a,b). Most frequently, an average number of 4 cytotoxic payloads are covalently attached to the $\mathrm{mAb}$. For cysteine ADCs for example, conjugates with zero, two, four, six or eight cytotoxic payloads per $\mathrm{mAb}$ are obtained, such that the average DAR is four [22]. A discrete distribution of odd and even drug loads is observed for lysine ADCs, as a result of the greater heterogeneity of the population. However, whereas lysine ADCs are assemblies maintained by covalent interactions between heavy and light chains (no reducing step that opens the disulfide bridges), cysteine ADC populations are mixtures of covalent and non-covalent compounds due to the presence of drugs at the interchain cysteine residues, making their analysis more complex. Analytical techniques and workflows therefore need to be adapted to obtain a clear and comprehensive understanding of the relationships between conjugation and the resulting product quality and heterogeneity. 
a) CysteIne conjugate
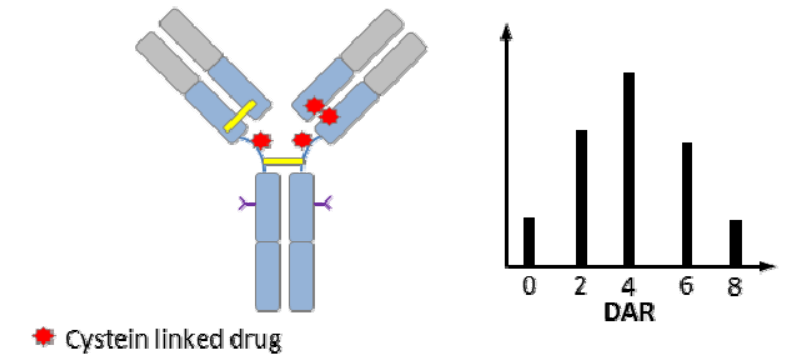

b) Lysine conjugate
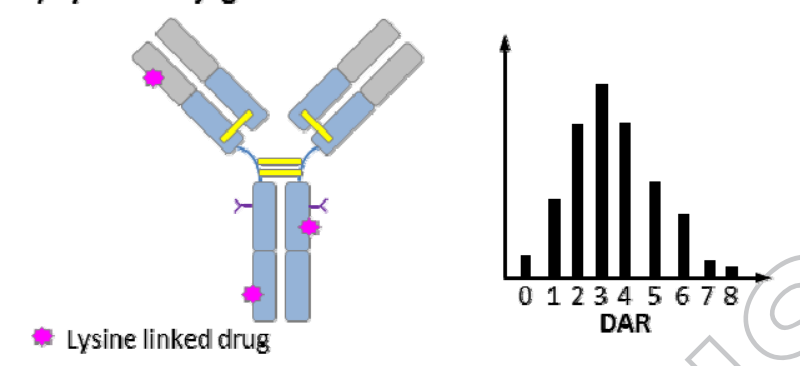

c) Site-specific conjugate

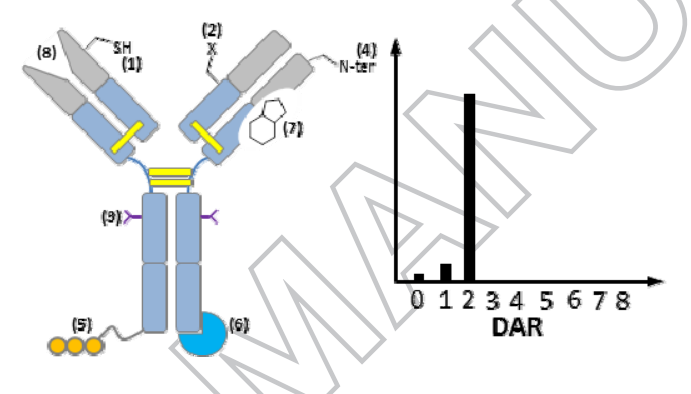

Figure 2. Schematic representation of the different types of ADCs : (a) cysteine hinge, (b) lysine hinge and (c) site-specific ADCs, and their corresponding drug-to-antibody ratio (DAR) distributions. The strategies that have been proposed to date for site-specific conjugation include (1) the covalent labeling of engineered cysteines; (2) inserting non-natural amino acids; engineering (3) the glycans or (4) the N-terminus of heavy and light chains, (5) adding engineered tags; (6) non-covalent binding using the affinity properties of Fc-binding domain; (7) photoaffinity labeling on the nucleotide binding site; and (8) the conjugation of a pharmacophore on the catalytic site.

\section{Main quality attributes for the characterization of ADCs}

The most important ADC characteristics required for process and formulation development, routine lot-release, and stability testing are the following [44]. The average DAR determines the amount of payload that can be delivered to the tumor cell and affects both the toxicity and safety of the ADC. The distribution of drug-loads (i.e. the fraction of antibodies containing zero, one, two, three, ... $\mathrm{n}$ drugs) is also an important characteristic since the different forms 
may have different toxicological and pharmacological properties. Because the cytotoxic drugs linked to antibodies are hydrophobic, conjugates are more likely to aggregate during manufacturing and storage. Furthermore, size variants - in particular those with high molecular weights (aggregates) - can modify the pharmacokinetics (that is, accelerate clearance) and reduce drug exposure. Finally, the concentrations of free drug (from cleavable linkers) or drug-linker compounds (from non-cleavable linkers) have to be controlled, since the unconjugated drug and its related products may pose serious toxicity and safety threats. Residual quantities of unconjugated drug or drug-related impurities may remain in the final product as a result of incomplete purification or of degradation during long-term storage. The proportion of unconjugated mAb must also be controlled.

\section{State-of-the-art analytical methods for the characterization of ADCs}

Average DAR measurements

A variety of methods have been used to measure the average DAR, the most appropriate depending on the properties of the cytotoxic drug and how it is linked to the antibody. The simplest method for estimating DARs is based on ultraviolet/visible (UV/Vis) spectroscopy [45], but the limitations of this approach (namely the UV photolability of calicheamicin, the lability of the linker, over-quantitation due to high concentrations of non-covalently bound drug) make confirmation using an orthogonal method necessary [46]. To measure the DAR of cysteine-linked drug conjugates, the most widely employed technique is hydrophobic interaction chromatography (HIC) [30,46-49]. This is performed under non-denaturing conditions at neutral $\mathrm{pH}$ with a gradient from high to low salt concentration. A low amount of organic modifier can also be included in the (low salt) mobile phase to improve the elution of mAbs loaded with lipophilic drugs. Under these conditions, each peak in the resulting chromatogram corresponds to an intact $\mathrm{mAb}$ species bound to a specific number of bound 
drug molecules (Figure 3b). Finally, native MS has recently emerged as an alternative technique in this context, as discussed below.

\section{Drug load distribution}

As for the average DAR, chromatography is the technique most commonly used to measure the drug-load distribution, sometimes coupled to MS. Chromatographic methods separate mAbs with different payloads based on the increased hydrophobicity imparted to the antibody upon conjugation with the drug linker. When the drug is connected to the antibody through inter-chain disulfide bonds, HIC (performed under non-denaturing and non-reducing conditions) is the benchmark method used to resolve the drug-load variants $[41,50]$. Reverse phase liquid chromatography (rpHPLC) (under reducing conditions) and sodium dodecyl sulphate capillary electrophoresis (SDS-CE, under both non reducing and reduction conditions)s can serve as orthogonal techniques to confirm the drug loading profile of cysteine ADCs $[47,48]$. When the conjugation is made through lysine amine residues, ion isolelectrofocusing (IEF) or imaged capillary isoelectrofocusing (icIEF) can be used to resolve the different ADC products. In this case, conjugation decreases the net positive charge of the mAb by one for each attached drug linker, if the drug-linker is itself uncharged. Finally, rpHPLC works well for ADC forms conjugated at specific sites (e.g., at inter-chain cysteines), but is less useful for more heterogeneous lysine-linked conjugates.

Size variants

These are typically determined by size exclusion chromatography (SEC), as is the case for intact mAbs. It is worth mentioning however that regular SEC using phosphate buffered mobile phase yields poor peak shapes for ADCs and an insufficient resolution between aggregates and monomeric ADC products [51]. This is probably the result of non-specific interactions between the cytotoxic drugs and the surface of the stationary phase. To overcome these difficulties, various organic modifiers have been added to the SEC mobile phase — such 
as $25 \%$ propylene glycol [52] or $10 \%$ DMSO [53] - to improve the shape of the resulting peaks. Alcohol-type organic modifiers may also prove valuable in improving the SEC performance of ADC products. For reduced fragments on the other hand-that is, variants with reduced charge namely light chains and heavy chains with different drug loads—-generic rpHPLC has successfully been used [50].

Free drug-linker quantification:

Concentrations of free drug molecules can be determined by enzyme-like immunosorbent assay (ELISA) and CE, but rpHPLC is currently considered the gold standard technique since it often provides the best selectivity, sensitivity and precision for cytotoxic drugs, which are typically lipophilic, low molecular weight compounds [54]. This approach can be followed by UV/MS, allowing the identification and quantification of various cytotoxic species released from the conjugate in stability testing studies.

In summary, the analytical methods most appropriate to characterize a given ADC strongly depend on the chemical properties of the linker, the cytotoxic payload and the attachment anchor (i.e. lysine or inter-chain cysteines) to the antibody. One should bear in mind that the assay (cationic exchange chromatography, for instance) used for the parent mAb may not always work for the corresponding ADC.

\section{A multi-level workflow for the comprehensive characterization of ADCs}

Dozens of $\mathrm{mAb}$ microvariants have been identified and reported in the literature, which differ in terms of their glycoform, charge, cysteine attachment, oxidation state, size, and gene sequence [55-58]. These microvariants and payloads-mAb conjugation both increase the structural complexity of the final drug substance [59], with over-deamidation typically occurring due to conjugation at basic pHs [59].

Figure 3 presents a general workflow for determining the critical quality attributes of ADCs. This multi-level approach allows the quality and heterogeneity of ADCs to be assessed 
at the level of the primary structure, but also probes the changes to the higher order structure arising from payload conjugation. The top level of the analysis involves mass measurements of intact $\mathrm{ADCs}(>150 \mathrm{kDa})$, typically using electrospray sources, either under native or classical denaturing conditions, which yield accuracies in the $30-100 \mathrm{ppm}$ range and reveal the glycoform heterogeneity, drug-load profile and the average DAR of covalent conjugates. If performed under classical denaturing conditions, this analysis is readily coupled to liquid chromatography (HPLC), enabling automated HPLC-MS experiments. On the other hand, native MS provides the drug or antigen binding stoichiometries. Top-down approaches can also be used to sequence intact mAbs, without proteolytic digestion [60-62].

The next (middle) involves the analysis of large ADC fragments $(25-50 \mathrm{kDa})$. The subunits obtained by reduction or enzymatic cleavage are amenable to LC-MS analysis, which with latest-generation spectrometers provides isotopic resolution and mass accuracies under 5 ppm. Improved fragmentation capabilities (using collision induced dissociation, and/or electron transfer dissociation) make middle-level approaches less demanding than intact mAb/ADC top-down analysis [63].

A peptide-level (bottom level) analysis of the ADC (0.7-7 kDa fragments) is still required if information on the primary sequence and/or the conjugation site are required. Classically, the cysteines are reduced and alkylated before the ADC is digested (with trypsin or endoproteinases such as Lys-C, Asp-N, Glu-C, or pepsin) and the resulting peptide fragments are identified through LC-MS/MS experiments and further searches in protein databases. Tandem mass spectrometry is also usually performed to sequence the peptides and to locate post-translational modifications such as glycosylation and the formation of disulfide bridges, notable at the conjugation sites.

The higher order structures (HOSs) of proteins (secondary, tertiary, and quaternary arrangements) are often responsible for their uniqueness and can govern their function, by 
preventing binding to antigens or Fcgamma and FcRn receptors for example. In this context, recent reports from the EMA or FDA have pointed out that knowledge of the HOSs of biopharmaceutical compounds is crucial for meaningful comparative studies between innovator products, biosimilars, and biobetters, but also, in case of ADCs, to assess the impact of conjugation on the overall structure of the mAb. As discussed below, this structural assessment can be performed by IM-MS and hydrogen/deuterium exchange coupled to MS (HDX-MS).

The fifth level of characterization presented here stems from the potential safety threats from residual drug-linker and related products in ADC batches, as mentioned above. These compounds can be quantified using the MS strategies for selected reaction monitoring (SRM) that have been developed for similar bioanalyses.

The relevance of this multi-level ADC characterization workflow is outlined in the next sections with two example applications, for BV and T-DM1.

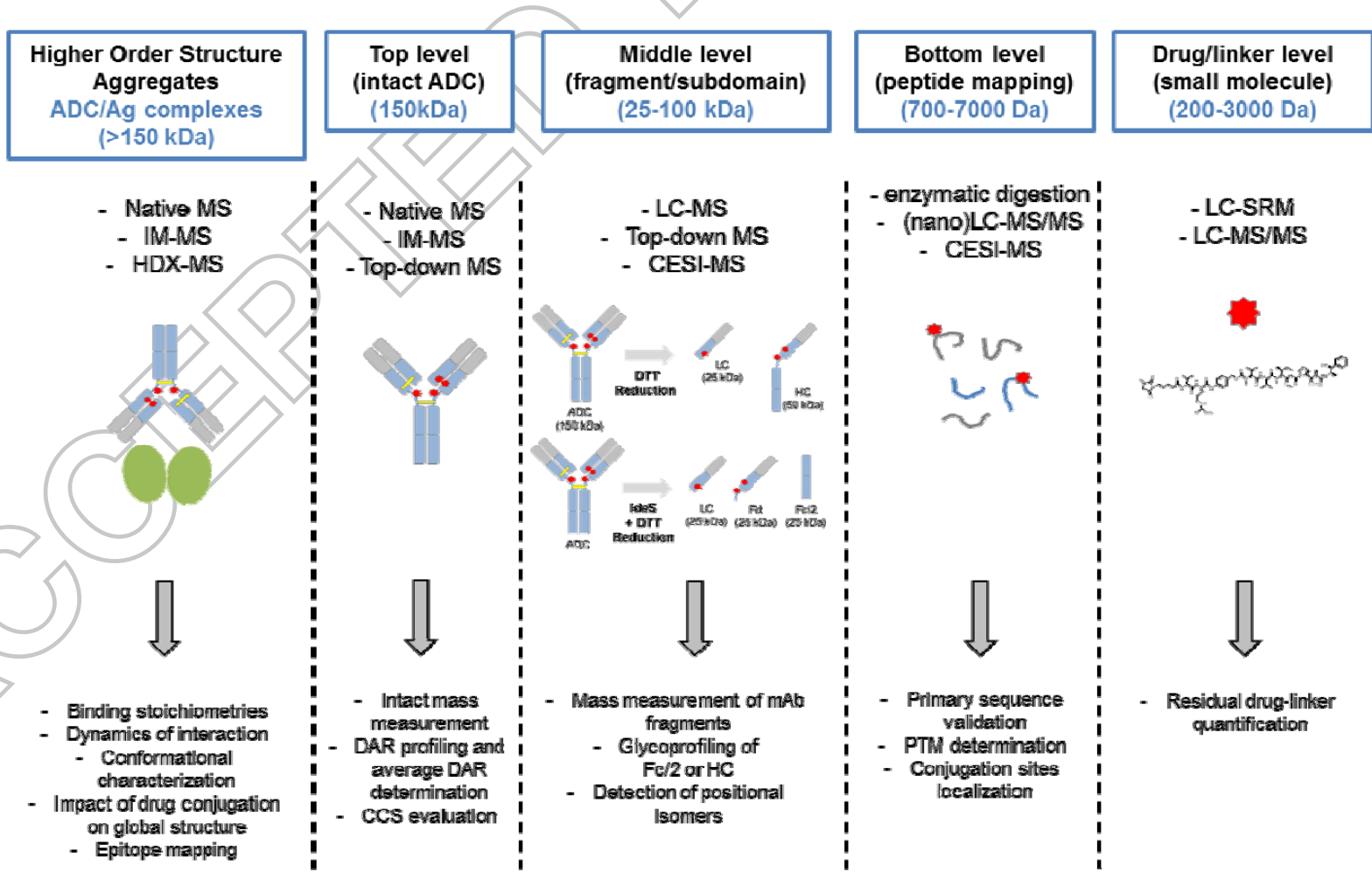


Figure 3: Multi-level workflow affording a comprehensive characterization of ADCs.

\section{Hinge-cysteine linked ADCs: BV as a case study}

Brentuximab vedotin is a potent ADC composed of the monoclonal antibody cAC10, which targets the CD30 antigen on Hodgkin lymphoma and systemic anaplastic large-cell lymphoma cells; a highly stable valine-citrulline linker; and a potent chemotherapeutic agent monomethyl auristatin E, which inhibits microtubule polymerization (Figure 1a). The conjugation strategy used for BV involves partially reducing the interchain disulfides of the anitbody prior to conjugation, yielding an ADC with 0-8 drug molecules toaded per antibody and an average DAR of four [22]. The presence of drug molecules at the interchain cysteine residues means that $\mathrm{BV}$ is a composite of covalently and non-covalently associated light and heavy chain subdomains (Figure 4a).

\section{Top level: intact BV analysis}

Classical intact mass measurements unden denaturing conditions are not appropriate for the characterization of cysteine-linked ADCs

The first step in the analysis of intact proteins is often a classical ESI-MS analysis under denaturing conditions, typically in an acidified $\mathrm{H}_{2} \mathrm{O}$ /acetonitrile solvent, either through direct infusion or by rpHPLC-MS analysis. For BV however, these harsh solvent conditions disrupt the non-covalent interactions between the light and heavy chain, hampering the detection of the intact ADC. As a result, the most intense MS signals usually arise from drug-linked antibody fragments, from both the heavy and the light chain, while minor signals are obtained from the free unconjugated $\mathrm{mAb}$ (Figure 4c) [64,65]. This suggests that denaturing MS analyses are inappropriate for the characterization of intact cysteine-linked ADCs and that a one-step determination of the drug load profile and average DAR is not possible for these compounds in classical denaturing conditions. 
Native and Ion Mobility MS as alternative methods for drug load profiling and average DAR measurements

Native MS has been used extensively for more than 25 years to study protein/ligand complexes from a variety of systems (for reviews, see $[66,67]$ ) but also to detect very large protein assemblies weighing several million Daltons $[68,69]$. It has more recently been shown to be a powerful technique for the analysis of intact mAbs [55,57,58,70-75]. ValliereDouglass et al. were the first to report the use of ESI-MS coupled to SEC under native conditions to record the mass of a cysteine-linked ADC and assess the relative distribution of drug-linked species [64]. The masses of the SEC-desalted ADCs were determined, using an ESI source and standard desolvation and ionization conditions, on a Q-TOF instrument for IgG1 mAbs conjugated with maleimidocaproyl-monomethyl Auristatin F (mcMMAF) and valine-citrulline-monomethyl Auristatin E (vcMMAE) at inter-chain cysteine residues. Elsewhere, Chen et al. employed nano- instead instead of conventional ESI in combination with limited enzymatic digestion to directly determine DARs [76]. Since then, several papers have used this technique to characterize the drug load distribution and to evaluate the average DAR for several cysteine linked ADCs, with results in good agreement with those obtained by HIC or UV/vis spectroscopy $[65,73,77,78]$. More recently, Valliere-Douglas described how native SEC-MS could be used in vivo for DAR measurements and to monitoring changes over time in the drug load distribution [79]. Debaene et al. performed an extensive native MS characterization of BV in comparison with its unconjugated form brentuximab, highlighting the efficiency and accuracy ( $<30 \mathrm{ppm}$ in routine analysis) of native MS on the latest highresolution Orbitrap instruments for drug distribution profiling and the determination of average DARs [65]. A relatively simple but efficient analytical workflow was described, consisting of desalting, optional deglycosylation, then native MS analysis of the ADC and semi-quantitative interpretation of the data obtained. The authors also highlighted the value of high-resolution native MS (Figure 4d) for one-shot ADC characterization, the limited overlap 
in the peak distribution obtained offering a unique description of intact glycosylated ADCs. Debaene et al. have also shown for the first time how native and IM-MS can be combined for the characterization of ADCs. The average DAR and the drug load profile were obtained through a direct extrapolation of semi-quantitative IM-MS data (Figure 4e). The native MS and IM-MS measurements were confirmed by HIC (Figure 4b) demonstrating the analytical potential of native MS strategies.

The main advantage of using native MS to characterize cysteine-linked ADCs lies in its ability to detect non-covalent associations of light and heavy chains, as these are not revealed by classical rpHPLC-MS methods. Furthermore, native MS data obtained on most quadrupole time-of-flight or orbirtrap instruments are sufficiently resolved to confirm the identity of the ADCs, and the relative distributions of the drug-loaded species derived from these data are in good agreement with those measured by HIC. Two additional advantages of native MS are its efficiency-with measurements completed in minutes rather than an hour for HIC - and the limited sample handling that is required, which limits the risk of artifacts appearing in the data. The process could indeed be automated by using one of several commercially-available SEC-native MS workflows.

Native Top Down MS of BV

Top-down MS is an emerging approach for the amino acid sequencing of intact proteins, providing valuable information on protein isoforms (proteoforms) [80,81], with the limited sample manipulation again reducing the occurrence of artifacts in the data. Top-down approaches have so far been largely performed on Fourier transform ion cyclotron resonance and Orbitrap instruments. While top-down analysis has been used to sequence mAbs [60$62,82-84]$, their (large) size means that currently achievable resolutions only provide $30 \%$ sequence coverage at best and incomplete sequencing of the complementarity determining 
regions [60]. The top-down (middle-level) analysis of smaller mAb fragments has proved more successful however, with 70\% sequence coverage having been achieved [63].

A recent interest in combining top-down proteoform analysis and structural characterization has lead to native top-down approaches being combined with ion mobility spectrometry either Q-TOF [85] or Orbitrap instruments [86]. Tandem native MS was successfully used by Dyachenko et al. for BV to show that drug conjugation takes place inhomogeneously at cysteine residues on both the light and heavy chains [86]. Thanks to the implementation of a high mass quadrupole on a high resolution Orbitrap instrument, precursor ions corresponding to one specific DAR were selected and then sequenced thanks to the MS/MS capabilities of the instrument. This located the conjugation site of the drugs in the antibodies and even revealed the positional isomers of the DAR 2 and DAR 6 species. 
a) Drug-load populations

1. Reduction

2. Conjugation

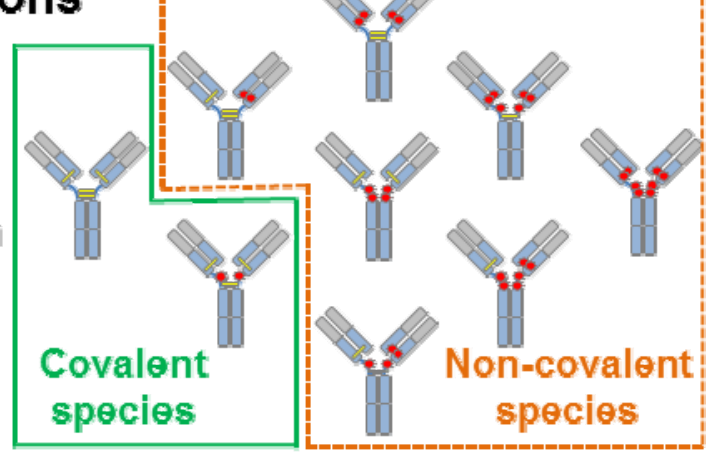

b) HIC

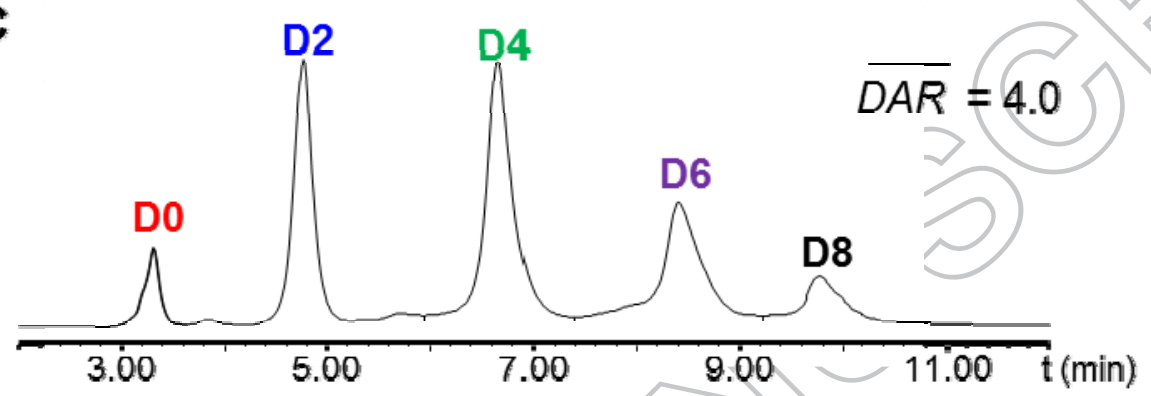

c) Denaturing MS

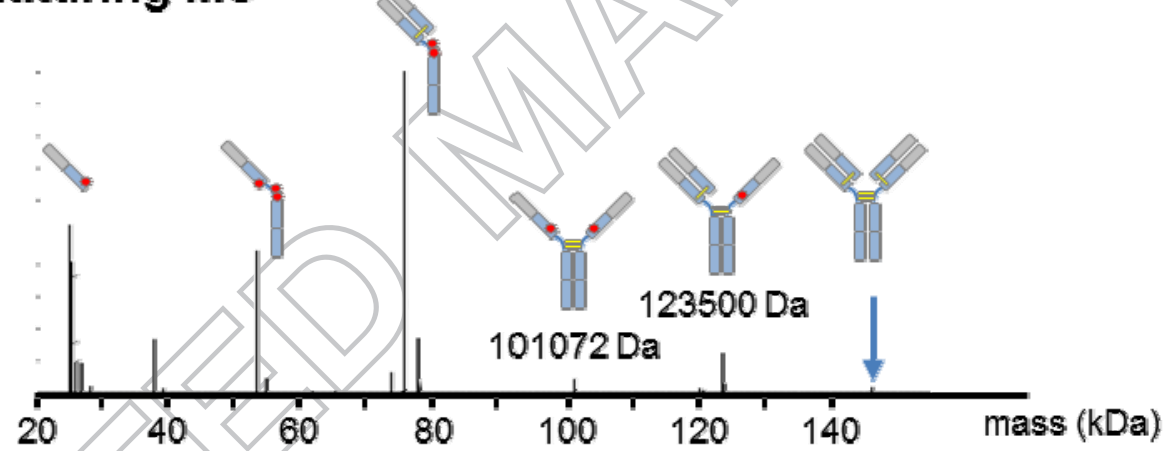

d) Native MS

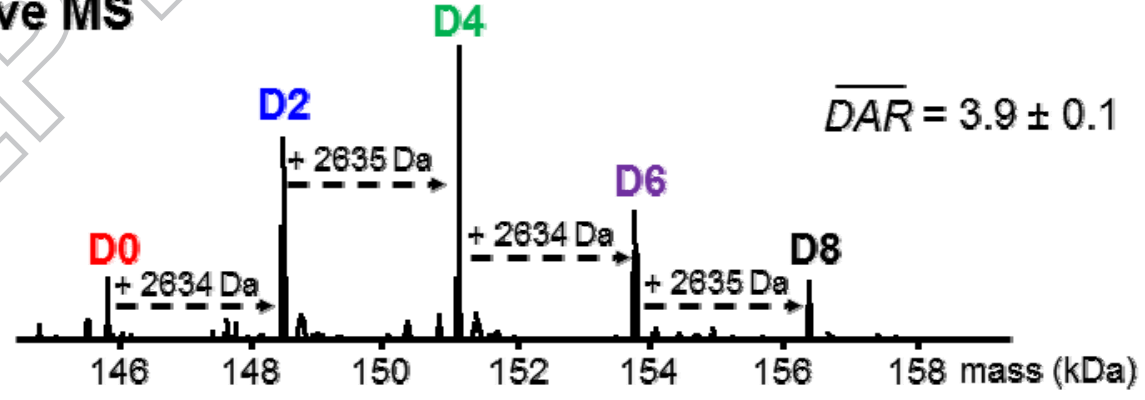

e) Native IM-MS

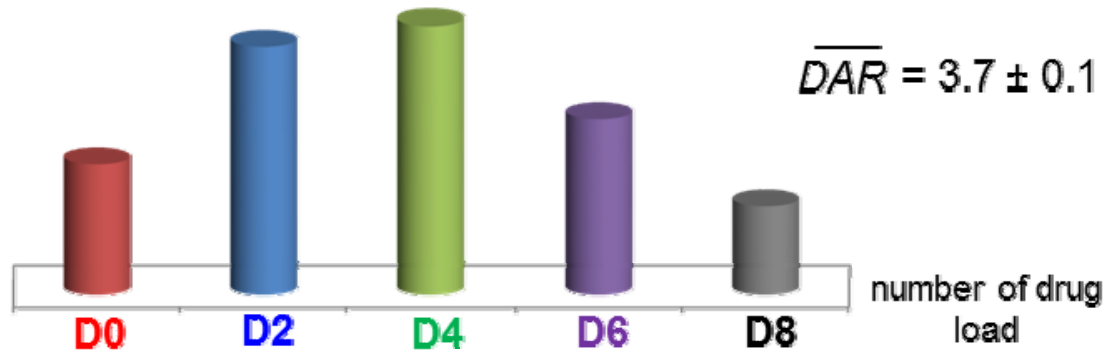


Figure 4. Top level analysis of brentuximab vedotin (BV). (a) Schematic representation of cysteine ADCs with a variable number of drug molecules per antibody. S-S bonds are represented by yellow lines while drug loads appear as red stars. (b) Reference hydrophobic interaction chromatogram revealing the drug loading profile and the average drug-to-antibody ratio (DAR). (c, d) Deconvoluted electrospray ionization mass spectra of deglycosylated BV (c) under classical denaturing conditions $\left(\mathrm{H}_{2} \mathrm{O}: \mathrm{ACN}: \mathrm{FA}\right.$ 50:50:1) and (d) under native

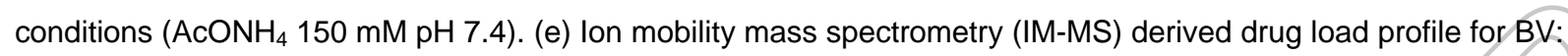
relative intensities of each drug load as a function of the number of drug molecules loaded onto the mAb.

\section{Middle level: BV subunit analysis}

Even if structural insights can be obtained for intact ADCs by native MS and IM-MS, the higher mass accuracy provided by the more straightforward rpHPLC-MS analysis of their subunits remains valuable. As for unconjugated mAbs, ADC profiles can be simplified by reduction [87] (yielding the light and heavy chains at $\sim 25$ and $\sim 50 \mathrm{kDa}$, respectively) or enzymatic treatments, such as N-deglycosylation (IgGZERO ${ }^{\circledR}$ or PNGase-F) [88], carboxypeptidase B digestion [87] or glutaminyl-peptide cyclotransferase treatment [89]. Smaller mAb fragments can also be generated by papain digestion (producing $\sim 50 \mathrm{kDa}$ $\mathrm{Fab} / \mathrm{Fc}$ fragments) or IdeS digestion (Fabricator ${ }^{\circledR}$, the immunoglobulin-degrading enzyme of Streptococcus pyogenes) followed by reduction with dithiothreitol (DTT, for Fc/2, LC and Fd fragments of $\sim 25 \mathrm{kDa}$, as illustrated in Figure 4 . This approach has the advantages of being fast (less than $2 \mathrm{~h}$ for the entire analysis including digestion and rpHPLC-MS analysis), informative, and inexpensive in terms of materials. Reduction experiments leading to individual light and heavy chains or IdeS treatment are to our knowledge currently mostly used for middle level analysis.

Characterization of BV subunits under denaturing and reducing conditions (middle level, 23-54 kDa fragments)

Reducing treatments are a routine way to divide the analysis of mAbs and ADCs into more manageable pieces. The drug loading profile and average DAR can then be obtained by rpHPLC with MS, as an orthogonal method to HIC [90]—as for the latter, rp-HPLC relies on 
differences in hydrophobicity. This middle-up strategy can be implemented on any current HPLC-MS instrumentation and is therefore available in most labs. Treatment of cysteinelinked ADCs with DTT or tris(2-carboxyethyl)phosphine (TCEP) fully reduces the remaining inter-chain disulfides and yields six species: light chains with zero or one drug molecule attached (L0 and L1), and heavy chains with zero, one, two or three drug molecules attached (H0, H1, H2 and H3). These species are stable in the denaturing organic solvent and can be successfully separated on a reversed phase column. The average DAR is obtained by calculating the weighting the proportions of the total integrated intensity under the light and heavy chain peaks according to their associated drug loads (Figure 5) [91,92].

a)

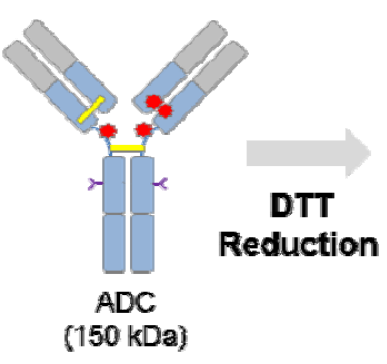

c)

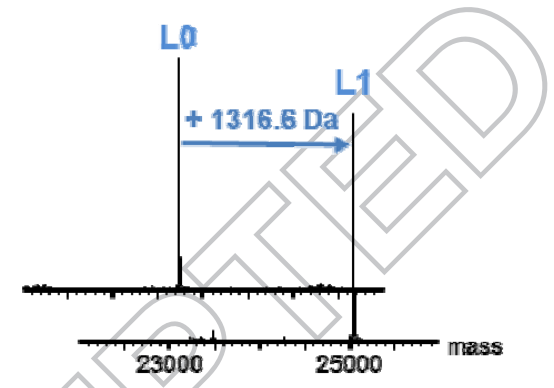

e)

Species Theoretical mass (Da) Experimental mass (Da) LO

1125094

HO 50328.6

H1 51644.4

$\mathrm{H} 2 \quad 52960.2$

H3 $\quad 54276.0$

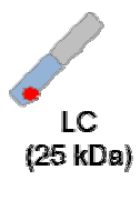

$25 \mathrm{kDa})$

23727.5

25044. 1

50327.0

51644.1

52960.4

54278.0

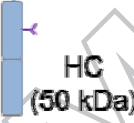

LC-MS

analysis
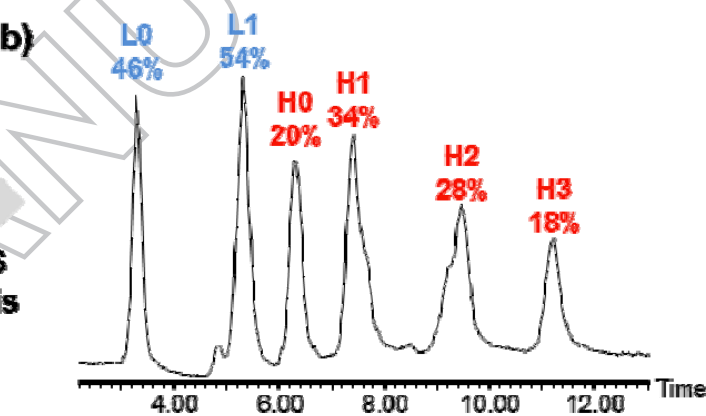

d)

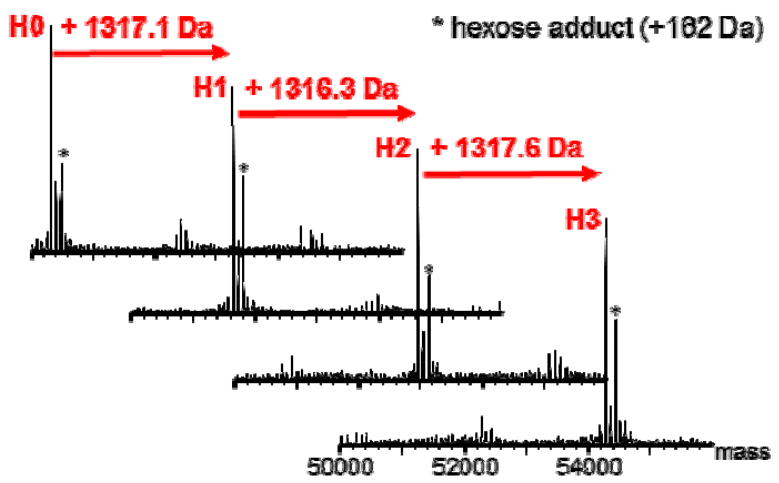

Delta (Da) Retention time (min) Distribution (\%) Average DAR

$\begin{array}{ll}0.8 & 3.32 \\ 0.0 & 5.33 \\ 1.6 & 6.33 \\ 0.3 & 7.42 \\ 0.2 & 9.48 \\ 2.0 & 11.22\end{array}$

4.0

Figure 5. Middle level characterization of brentuximab vedotin (BV) after reduction. (a) Schematic representation of the workflow for middle-level analysis. (b) Reverse-phase high-pressure liquid chromatogram of BV fragments obtained after reduction with dithiothreitol and alkylation with idoacetic acid. (c, d) Deconvoluted 
mass spectra of (c) the light- and (d) the heavy-chain fragments of BV. (e) Table listing the masses, proportions, and average drug-to-antibody ratios of the different BV fragments, as measured from the corresponding peak areas.

Characterization of BV subunits after enzymatic cleavage (middle-level, 23-28 kDa fragments)

Downsized $\mathrm{mAb}$ or ADC fragments an also be obtained by limited proteolytic cleavage under non-denaturing conditions in the hinge region of the heavy chain, yielding Fab or (Fab' $)_{2}$, and Fc fragments, whose reduction (with DTT) produces even smaller fragments of approximately $25 \mathrm{kDa}$ : the light chain and the two halves of the heavy chain $(\mathrm{Fc} / 2$ and $\mathrm{Fd})$. Formerly conducted with proteases with a limited specificity, such as papain [93], pepsin [94], and endoprotease Lys-C [95], the enzymatic cleavage for middle level analyses is mostly conducted using IdeS, a bacterial protease that specifically cleaves IgGs under the hinge region [96]. The potency of IdeS has been demonstrated for cysteine-linked ADCs on an antibody-fluorophore conjugate [88]. A rapid IdeS- and rpHPLC-MS-based procedure was also recently employed by Firth et al. for the characterization of two auristatin ADCs [97]. With IdeS, a complete middle level characterization can be completed within a few hours, providing the primary sequence and the glycoprofiles of the Fab and Fc fragments. The data can also be used for biosimilar comparability studies and Fc-fusion protein studies. More recently, IdeS digestion has been shown to be preferable to DTT treatment for the characterization of BV, the LC fragments being better separated in the subsequent rpHPLCMS analysis allowing the identification of positional isomers (Figure 6) [92]. In addition to the seven expected major peaks (from the Fc/2, L0, L1, Fd0, Fd1, Fd2 and Fd3 fragments) two minor satellite peaks with identical masses are observed close to those from Fd1 and Fd2 (Figure 6) and tentatively assigned to positional isomers. This was confirmed by peptide mapping with nanoLC-MS/MS following the digestion with endoprotease Lys-C of isolated fragments, as discussed below. 
a)

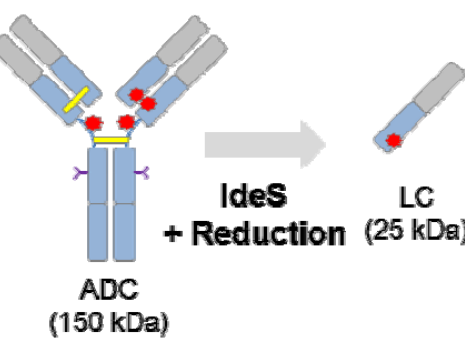

c)

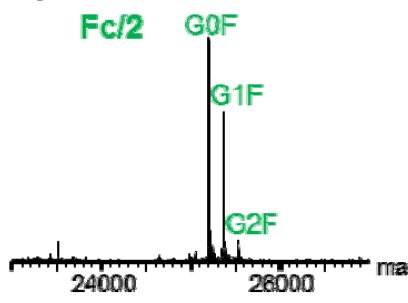

d)

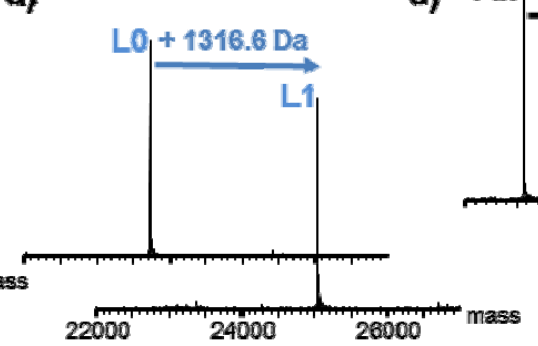

b) $\mathrm{Fe} / 2$
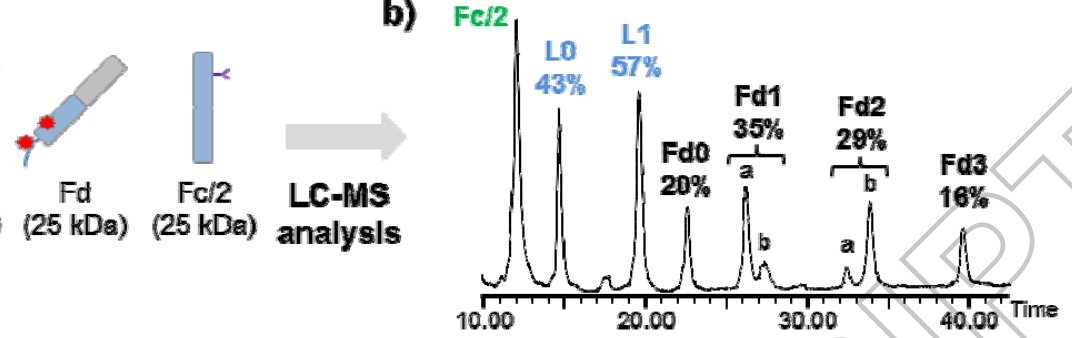

+1316.5 Da

f)

Species Theoretical mass (Da) Experimental mass (Da) Delta (Da) Retention time (min) Distribution (\%) Average DAR

\begin{tabular}{cccccc}
\hline Fc/2 (G0F) & 25203.6 & 25203.9 & 0.3 & 11.93 & - \\
Fc/2 (G1F) & 25365.8 & 25365.9 & 0.1 & 11.93 & - \\
Fc/2 (G2F) & 25528.0 & 25528.1 & 0.1 & 11.93 & - \\
L0 & 23728.3 & 23727.8 & 0.5 & 14.62 & 43 \\
L1 & 25044.1 & 25044.4 & 0.3 & 19.55 & 57 \\
Fdo & 25142.3 & 25141.8 & 0.5 & 22.53 & 20 \\
Fd1a & 26459.1 & 26458.3 & 0.8 & 26.15 & 35 \\
Fd1b & 26459.1 & 26458.3 & 0.8 & 27.40 & \\
Fd2a & 27774.9 & 27774.5 & 0.4 & 32.43 & 29 \\
Fd2b & 27774.9 & 27774.6 & 0.3 & 33.85 & 16 \\
Fd3 & 29091.7 & 29091.9 & 0.2 & 39.57 &
\end{tabular}

Figure 6. Middle level characterization of brentuximab vedotin (BV) after digestion with IdeS (the immunoglobulin-degrading enzyme from Streptococcus pyogenes). (a) Schematic representation of the workflow for middle level analysis. (b) Reverse-phase high-pressure liquid chromatogram of BV fragments obtained after/des cleavage and dithiothreitol reduction. (c-e) Deconvoluted mass spectra of (c) the light chain, (d) the Fc/2 and (e) the Fd fragments of BV. (f) Table listing the masses, proportions, and average drug-toantibody ratios of the different BV fragments, as measured from the corresponding peak areas.

Bottom level: peptide mapping of $\mathrm{BV}$ and the characterization of positional isomers (0.3-7 kDa fragments)

Following denaturation, reduction, and alkylation of the Cys residues, mAbs or recombinant proteins are commonly digested with trypsin or endoproteinases such as Lys-C, Asp-N, or Glu-C. Samples can be prepared automatically thus facilitating multiple analyses. The extra 
hydrophobicity from the drug molecule means however that peptide mapping is more difficult for cysteine-linked ADCs than for mAbs, especially for species bearing two or more conjugated drugs [44]. As a result, ADCs have seldom been characterized at the peptide level. Junutula et al. describe the site-specific conjugation of vcMMAE to an antibody through engineered cysteine substitutions on the light and heavy chains resulting in only tree isoforms with zero, one or two drug molecules and no positional isomer [30]. The tryptic peptide mapping of these conjugates, with LC-MS detection, identified four drug-conjugated peptídes via a characteristic in-source fragmentation ion $(\mathrm{m} / \mathrm{z}=718.5)$ that is observed in all the mass spectra of molecules containing vcMMAE. The peptides were all found to be fragments of either complete or partial tryptic cleavage around the engineered cysteines. More recently, Janin-Bussat et al. reported an improved BV peptide mapping protocol allowing the drugloaded peptides to be identified by LC-MS analysis [92]. Because of the hydrophobicity of the drug, all steps of the peptide mapping protocol were adjusted to maintain the hydrophobic drug-loaded peptides in solution and enable their unambiguous identification by LC-MS. In particular, solvents were added immediately before (10\% acetonitrile) and after (40\% isopropanol) the enzymatic digestion step. The resulting data showed that as expected, the drug molecules were linked to the inter-chain cysteines of the heavy and light chains. Furthermore, after IdeS digestion and reduction of the ADC, the positional isomers were identified for the first time by LC-MS/MS analysis, unambiguously demonstrating that when only one drug molecule binds, the link forms on Cys220 of the L15 peptide of the heavy chain. Conversely, when two drug molecules attach to the heavy chain, they bind preferentially to Cys226 and Cys229 of the L16 peptide [92]. Interestingly, Birdsall et al. recently demonstrated a rapid on-line method based on MS and multidimensional chromatography, which allowed them to confirm the structure of a cysteine-conjugated ADC base on an IgG1 [98]. The HIC peaks in the $1^{\text {st }}$ dimension were successfully assigned to specific subunits via 
MS following dissociation under denaturing reverse-phase conditions (the $2^{\text {nd }}$ dimension). Elsewhere, a comparative characterization by LC-MS of the cysteine-linked conjugation profiles of IgG1 and IgG2 ADCs has been performed by Wiggins et al. [99]. The results demonstrated that IgG1 monoclonal antibodies favor conjugation to the cysteines between the light and heavy chains, whereas IgG2s link preferentially to cysteines in the hinge region, Finally, as an example, application of new enzymes that should also prove valuable for the characterization of ADCs, Srzentic et al. used secreted aspartic protease 9 (SAP9) from C. albicans, which generates 3-4 $\mathrm{kDa}$ peptide fragments, and bottom-up proteomics to achieve near $100 \%$ sequence coverage on a mAb in a single LC-MS run [100].

\section{Lysine linked ADCs: T-DM1 as a case study}

Trastuzumab emtansine is the third ADC to have received market approval and is indicated for the treatment of HER2-positive breast cancer patients [23]. Trastuzumab (Herceptin ${ }^{\circledR}$ ) which mitigates the effects of HER2 overexpression, is coupled to a maytansine derivate (DM1) which inhibits the elongation of microtubules. In contrast with BV, T-DM1 can be conjugated without reduction, so that T-DM1 exists only as covalent species. Zero to eight drug molecules are attached with an average DAR of 3.5 [101].

This suggests that T-DM1 is easier to characterize than BV; however, the methods (primarily $\mathrm{HIC}$ ) that are used to determine the average DAR for cysteine-conjugates are difficult to adapt for the analysis of lysine-conjugates. Indeed, the high degree of heterogeneity that characterizes lysine ADCs, which have at least 40 lysines available for conjugation, makes UV/vis spectroscopy and MS approaches more suitable [45]. The systems amenable to the former are rather few in number as i) the drug must be detectable by $\mathrm{UV} / \mathrm{vis}$ wavelengths; ii) the drug and $\mathrm{mAb}$ must have different maximum absorptions and iii) the absorption of light should not influence the interaction between the drug and the mAb. 
Lysine-linked conjugate species with different drug loads can be resolved by icIEF, but the presence of charge-associated variants of the antibody intermediate and any cross-linking or differences in the UV responses of the differentially drug-loaded forms may prevent quantification $[102,103]$.

Mass spectrometry based techniques are therefore pivotal for the characterization of lysine-conjugated ADCs [25,90,104-106]. Several papers have demonstrated the potential of LC-MS and SEC-MS to respectively measure the average DAR and drug load distribution on maytansin-conjugated mAbs $[25,90,101]$. Marcoux et al. have recently demonstrated the ability of native MS and native IM-MS to characterize lysine-conjugated ADCs, notably TDM1 [78]. These authors first compared the potential of native MS yersus denaturing MS for the determination of average DARs and drug load distributions. The presence of highly charged species of T-DM1 leads to charge state overlap, especially under denaturing conditions, such that only species with DARs of zero to seven (at best) can be observed (Figure 7a). High-resolution native MS is nonetheless beneficial as it reveals DAR 8 species at the intact protein level (Figure $7 \mathrm{~b}$ ). Charge reduction using imidazole also allows the detection of all the species from DAR 0 to DAR 8, and the average DAR thus obtained for T$\mathrm{DM} 1,3.5 \pm 0.1$, is in agreement with the value reported by Lazar et al. [101]. As for cysteine ADCs, native IM-MS highlights the increase in heterogeneity that occurs when trastuzumab is conjugated with DM1 (Figure 7c). The drug distribution profile obtained and the average $\operatorname{DAR}(3.4 \neq 0.2)$ are consistent with values obtained from other MS methods. Huang et al. have also illustrated the potential of IM-MS in a routine rpHPLC-MS analysis of an IgG1 lysine conjugate, showing in particular how a "cleanup" step significantly improves the signal-to-noise ratios in the mass spectra of the intact ADC, and increases the accuracy of the associated DAR measurements [107]. 
To assess drug loading heterogeneity and the location of the drug conjugation site, Gautier et al. have reported the combined used of native MS and bottom up proteomics for the characterization of IgG1 and IgG4 ADC-like components, using N-hydroxysuccinimide-based tandem mass tags as a mimic for ADC conjugation. Native MS allowed the detection and quantification of up to 69 conjugates while bottom up proteomics revealed some very reactive lysine conjugation sites, or "hot spots" [108].
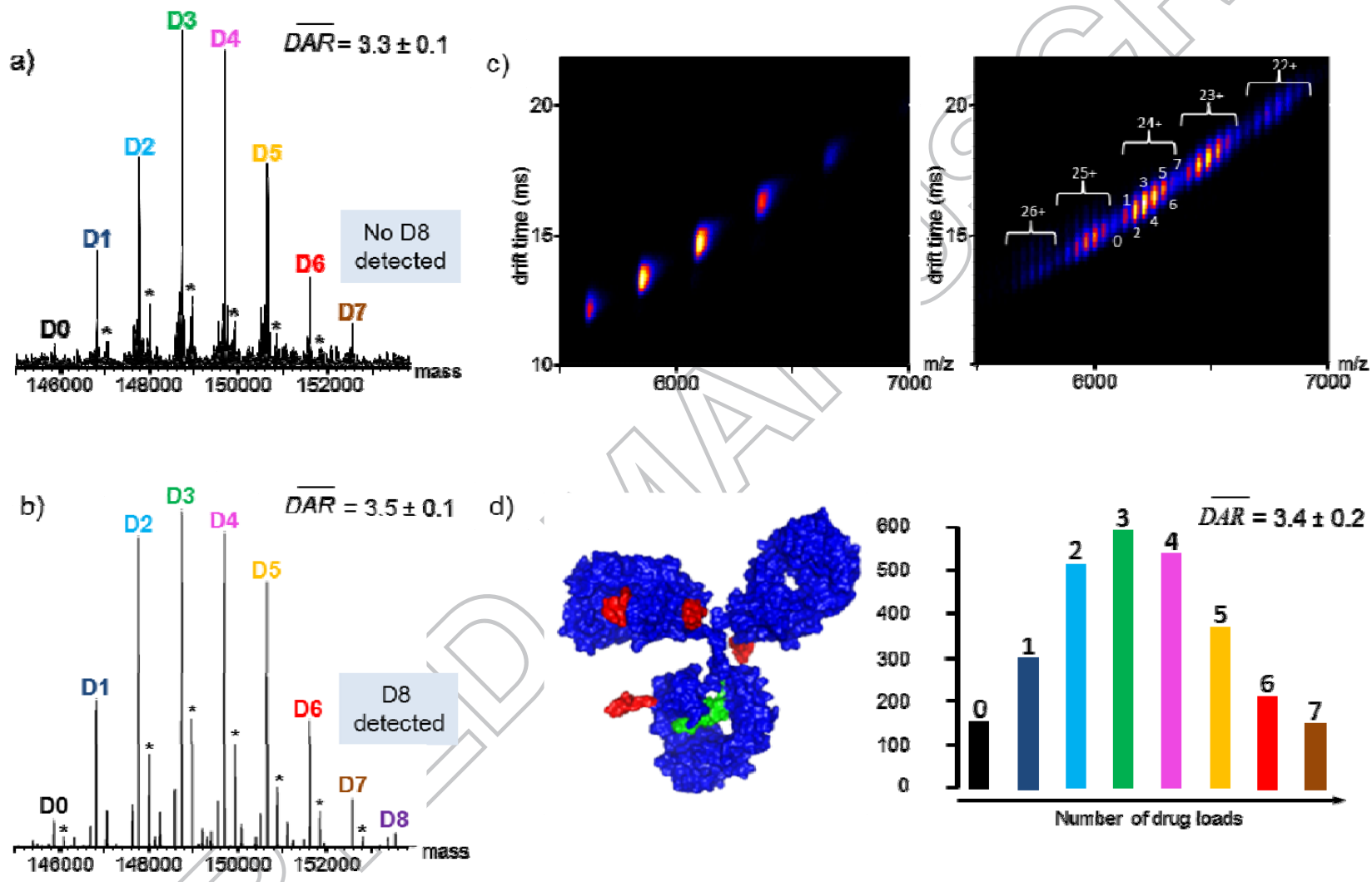

Figure 7. Top level analysis of trastuzumab emtansine (T-DM1). (a) Deconvoluted electrospray ionization mass spectra obtained under denaturing conditions $\left(\mathrm{H}_{2} \mathrm{O}: \mathrm{ACN}: \mathrm{FA} 50: 50: 1\right)$ by direct infusion on a Q-TOF instrument of T-DM1 after deglycosylation. (b) Deconvoluted electrospray ionization mass spectra obtained under native conditions $\left(\mathrm{AcONH}_{4} 150 \mathrm{mM} \mathrm{pH} \mathrm{7.4)}\right.$ by direct infusion into a high resolution Orbitrap extended mass range instrument (nominal resolution: $17.5 \mathrm{k}$ ) of T-DM1 after deglycosylation and charge reduction with $40 \mathrm{mM}$ imidazole. (c) Ion mobility-mass spectroscopy (IM-MS) driftscope plots obtained for trastuzumab (left) and T-DM1 (right) highlighting drug load heterogeneity. (d) Molecular model of trastuzumab conjugated with four DM1 payloads and drug load profile of deglycosylated T-DM1 obtained by IM-MS. The asterisks indicate 1220 Da linker adducts. 


\section{Site specific ADCs}

As discussed and illustrated above, the three market-approved ADCs, and most of those under clinical trial are controlled mixtures of molecules. In 2008, Junutula et al. reported the design and production of a new class of homogeneous ADCs, thiomab-drug conjugates (TDCs), prepared by conjugation to engineered IgGs containing additional exposed cysteine residues whose position were identified using phage display methods [30]. After reducing the blocked cysteine residues and interchain disulfides, the latter were regenerated and maleimide reagents were conjugated to the reactive cysteine thiol group to generate site-specifically modified ADCs with $92.1 \%$ of the population having two loaded molecules and only $0.2,3.3$, and $4.3 \%$ having zero, one, or three, respectively (average DAR $=2.0$ ), as assessed by rpHPLCMS. Importantly, these TDCs are better tolerated at higher doses in animals, indicating that their therapeutic index is higher than that of existing ADCs. A plethora of other site specific techniques have subsequently been reported, as recently reviewed (amongst others) by Panowksi et al. [109], Agarwal and Bertozzi [110] and Dennler et al. [111], and summarized in Figure 2c. Interestingly, Junutula and colleagues published another paper, in 2012 [112], indicating that their first-generation TDCs were not very stable in the blood stream. Thanks to MS, the degradation mechanism was shown to be a retro-Michael reaction, resulting in the transfer of the drug linker to circulating molecules bearing free thiol groups such as albumin and glutathione [113]. In addition, based again on mass shift measurements, succimide ring hydrolysis was shown to stabilize the drug linker on the antibody was observed, with a mass increase of $18 \mathrm{Da}(\mathrm{H} 20)$. This ring opening was correlated with a positively charged local site. Succinimide hydrolysis has since been employed to improve the stability (and hence the safety) of several ADCs [114]; different strategies have been adopted, notably the processfavored mild method based on a basic $\mathrm{pH}$ treatment [115], but also self-hydrolyzing maleimides [114] and electron withdrawal N-substituents [116]. 
Alternatively, the specific conjugation to N-glycans of IgG (at Asn297) can be achieved by periodate oxidation of fucose followed by hydrazone condensation, metabolic incorporation of 6-thiofucose and then maleimide conjugation [117]. The process is completed using specific enzymes such as enzymatic transfer of galactose and sialic acid followed by periodate oxidation and oxime condensation, enzymatic transfer of galactose and 9-azidosialic acid followed by $\mathrm{Cu}$-free click reaction, enzymatic removal of terminal galactose followed by enzymatic transfer of GalNAz and $\mathrm{Cu}$-free click reaction [118]. Each step in the reaction can be monitored by LC-MS, which can then also be used to assess the structures [119], and the glyco-profiles [120].

Conjugation can also be achieved through the incorporation of non canonical aminoacids - such as p-acetylphenylalanine sometimes with an azido-lysine derivative - and then oxime condensation and $\mathrm{Cu}$-free click chemistry. The cell-free incorporation of $\mathrm{p}$ azidomethylphenylalanine followed by $\mathrm{Cu}$-free click chemistry, and the incorporation of selenocysteine followed by mild reduction and alkylation have also been described [121]. As for other site-specific conjugation techniques, the choice of attachment site may affect the stability of the resulting ADCs [39].

Conjugation methods based on the enzymatic modification of peptide tags have also been explored extensively. One method involves glycosidase treatment— to access Q295 (and N297Q mutants prior to the transglutaminase-mediated conjugation of aminefunctionalized small molecules or drugs to engineered LLQGA sites [33]. The sortasemediated conjugation of a glycine-functionalized chelator near the C-terminus of a singlechain antibody ( $\mathrm{scFv})$ and the formylglycine generating enzyme-mediated conversion of cysteine to formylglycine followed by hydrazino-Pictet-Spengler ligation have also been successfully generated [122]. 
One of the caveats of these site-specific approaches is that many commonly used bioorthogonal reagents do not react stereospecifically and typically yield at least two diastereomeric products, resulting in several regioisomers. In addition, since these conjugation methods are applied to heterogeneous antibodies (e.g. glyco- and charge-variants), the homogeneity of the final products depends on the resolving power of the analytical and structural methods employed [58].

\section{ADC higher order structure and aggregates}

The HOS of a protein, namely its secondary, tertiary and quaternary assemblies, is often essential for its function [123,124]. While disruption of the native fold can also impact the function of the molecule, the main immunogenic consequences of misfolded proteins stem from their propensity to aggregate $[125,126]$. In solution, $\mathrm{mAbs}$ and ADCs tend to selfassociate, forming a range species from oligomers to visible particles. Changes in the HOS of ADCs can alter their quality attributes, and thereby their safety, efficacy and pharamacokinetics. A requirement of regulatory agencies is therefore that the effects of manufacturing, storage and delivery on the HOSs of mAbs and ADCs be well characterized [127].

Determining the HOSs of proteins using analytical biophysical techniques is still challenging however. The classical methods for protein structure determination, namely X-ray diffraction (XRD) and nuclear magnetic resonance (NMR) [128], provide atomic resolution but can only tackle relatively small proteins, let alone protein assemblies, and consume large amounts of biological material. For mAbs and ADCs furthermore, the flexibility of the hinge region hinders the growth of crystals (for XRD) and complicates NMR analyses. So far, only a small number of mAb crystal structures have been published [129] and no 3D structure of an ADC has been solved. In this context, lower-resolution methods, notably those based on MS (see Figure 8 and the following sections), can provide valuable insights. 


\section{Native MS and IM-MS}

Native MS has been used to reveal the presence of mAb multimers (dimers, trimers, tetramers) [130] and to study the structural dynamics of IgG4 [74]. As non-covalent interactions are maintained, a single native MS analysis can be used to both characterize the ADC (average DAR, drug load distribution, proportion of unconjugated drug molecules) and check for the presence of multimers. Native MS has also been used to unambiguously determine antibody/antigen binding stoichiometries [131] and to monitor the dynamics of IgG4 Fab-arm exchange [74]. Furthermore, Haberger et al. recently reported a linear correlation between results obtained by quantitative native $\mathrm{MS}$ and IgG-FcRn functional analysis, a larger amount of higher order complexes being detected in the absence of methionine oxidation in both cases [132].

The structural analysis of proteins can also be facilitated by IM-MS [133-136]. In this approach, ions are separated based on their collisional cross section (CCS) as they drift through an inert buffer gas in an electrical field, providing information on the charge and conformation of a protein complementary to the associated mass data. The measured drift times can be converted into CCSs, which correspond to the averaged rotational projection on a 2D space of the biomolecule 3D structure. IM-MS adds an additional level of conformational characterization to that provided by native MS. The analysis of the HOSs of mAbs by IM-MS remains a relatively novel endeavor [56,57,72,74,131,137-140].

To our knowledge, only three publications have so far reported the use of native IMMS for the characterization of ADCs $[65,78,107]$. Debaene et al. extensively analyzed the higher order structure characterization of BV by IM-MS [65], comparing the heterogeneity of BV with that of unconjugated brentuximab. Five different species were observed, having zero to eight drug molecules attached but similar gas phase conformations-viz. CCSs of $71.1 \pm$ $0.1 \mathrm{~nm}^{2}$ and $68.1 \pm 0.1 \mathrm{~nm}^{2}$ for the DAR 8 and DAR 0 species, respectively. The constant shift 
in CCS between two consecutive even drug conjugates points to a small increase in mass rather than to structural changes and indicating that the conformation of the protein is not drastically altered upon binding.

Similarly, Marcoux et al. performed a global conformational analysis of T-DM1 [78] and presented the first attempt 3D model of an ADC based on the structure of human $\operatorname{IgG1}$ and the Fab structure of trastuzumab (PDB IDs $1 \mathrm{HZH}$ and $1 \mathrm{~N} 8 \mathrm{Z}$, respectively). The CCSs derived from IM-MS were found to be slightly higher than those estimated from the masses of the species, but considerably lower than those expected from molecular modeling. These discrepancies, also observed for unconjugated $\mathrm{mAb}$ and attributed to a collapse of T-DM1 in the gas-phase $[65,138]$, contrast with the results obtained for BV, for which the values calculated assuming spherical proteins match those measured by IM-MS. This suggests that the surface-exposure of lysine-linked payloads makes them more susceptible to gas-phase collapse than equivalent payloads in cysteine-linked conjugates. Each drug-loading event induced a CCS increment of $\sim 25 \AA^{2}$, which matches the binding contribution expected from the mass of a single DM1 molecule $\left(\sim 100 \AA^{2}\right)$. This suggests that the conformational changes that occur in trastuzumab upon drug conjugation are very slight.

As mentioned above in the discussion of analytical methods for T-DM1, Huang et al. [107] have also employed IM-MS to perform DAR measurements. The protein drift time was used as a second dimension to monitor the changes in DAR in lot-to-lot analyses of both a site-specifically and randomly conjugated ADC.

Although it remains an emerging technique native IM-MS offers a simple and direct means to assess the size and shape of ADCs and thereby obtain a rough conformational picture. The examples above also illustrate how IM-MS can be used to identify all the species with different drug loads present in solution and study the effects of drug conjugation on the global conformation of the $\mathrm{mAb}$. In the medium term, the development of molecular 
dynamics algorithms able to cope with $>150 \mathrm{kDa}$ proteins should facilitate the structural interpretation of IM-MS data on mAbs and ADCs.

\section{HDX-MS}

Technical developments over the past 10 years have brought HDX-MS to the forefront of methods for the structural characterization of proteins. Indeed, HDX-MS now fills a niche in their therapeutic analysis, notably for epitope mapping and comparability studies $[141-147]$, but also in the investigation of $\mathrm{mAb} / \mathrm{Fc}$ receptor interactions [148]. Papers have also been published on the use of HDX-MS to study mAb aggregation [149-151], to optimize formulation stability [152], to monitor reversible mAb self-assembly [153], and to characterize the structural effects of glycosylation or chemical modifications on mAbs [154$157]$.

In a typical HDX-MS experiment, the protein of interest is deuterated over different lengths of time, after which the reaction is quenched under strong acidic conditions at low temperature $\left(0{ }^{\circ} \mathrm{C}\right)$. A classic proteolytic digestion is then performed using pepsin, which cleaves non-specifically but with a high reproducibility and under acidic conditions. The digested peptides are then analyzed by $\mathrm{rpLC}-\mathrm{MS}$ to assess their level of deuteration. An additional level of IM separation can help to assign the peptides and increases the sequence coverage (Figure 8). HDX-MS can be performed on proteins or assemblies with only a dozen picomoles of material (vs. milligrams for crystallography) and no size limitations in theory (in practice HDX-MS analysis of assemblies up to $300 \mathrm{kDa}$ were reported $[151,158]$ ).

Its other main strength is its ability to monitor any structural changes that occur upon

labeling in solution.

A field of application of HDX-MS not mentioned above is the study of mAb HOSs. Circular dichroism (CD), fluorescence spectroscopy and differential scanning calorimetry (DSC) are the methods most frequently used at present $[159,160]$. For instance, Guo et al. 
reported similar $\mathrm{CD}$ profiles for vcMMAE and the isolated mAb (MMAE), suggesting that the secondary and tertiary structures of cysteine-linked ADCs do not differ significantly from those of the parent mAb [161]. However, a key limitation of these biophysical techniques is that the information obtained is an average both over all the protein conformers in solution and across the sequence of the protein. The better spatial resolution offered by HDX-MS at the peptide level at least and at the amino acid level when combined with electron transfer dissociation) and its capacity to generate dynamic data for solution samples, makes HDX-MS an attractive alternative to probe the HOSs of mAbs and ADCs.

By comparing the deuterium uptake plots of the conjugated and unconjugated MMAE, Pan et al. showed that almost $90 \%$ of the primary sequence of vcMMAE has the same HDX kinetics as its parent $\mathrm{mAb}$, the only differences occurring for the two peptides in the $\mathrm{Fc}$ domain which have faster kinetics after conjugation [162]. This suggests that vcMMAE and MMAE have very similar conformations and dynamics in solution. A detailed comparison of the HDX-MS profiles of the ADC and its corresponding TCEP-reduced mAb indicated that the partial reduction of the $\operatorname{IgG1}$ interchain disulfides induced some minor, local structural differences in the conformation and dynamics of the mAb Fc region. However, alkylation of the reduced cysteine residues by the drug molecule does not further impact the local structural of the domains where the differences were observed [163]. The same group also used HDXMS to demonstrate that site specific conjugation on engineered cysteine has only minor structural effects on other ADCs (with pyrrolobenzodiazepine or mcMMAF as the conjugated drug), the only difference being a slightly higher deuterium uptake after conjugation in the vicinity of the mutation ( ${ }_{26} \mathrm{VDSV}$ ) because of the destabilization of hydrogen bonds between $\mathrm{C}_{239}$ and $\mathrm{V}_{264}$ induced by the drug-linker [164].

Since HDX-MS can provide information the conformations and dynamics of ADCs in solution - information that would be more difficult to obtain via other analytical methods - it 
should become a technique of choice for ADC comparisons, the optimization of conjugation sites, and the choice of the best drug-linkers in the development of therapeutic candidates.

\section{Aggregation}

As aggregation is the main cause of drastic decrease in the therapeutic efficiency of proteins, with potentially deleterious effects on patient health, a crucial characteristic of ADCs is the proportion of aggregates in the final product [124]. Both mAbs and ADCs can become unstable during conjugation $[160,165,166]$ or under environmental stress (from changes in temperature or ionic strength, exposure to light, etc.). However, aggregation is more common for ADCs, because the solubility of the $\mathrm{mAb}$ is reduced upon conjugation to hydrophobic drug-linkers $[44,161]$.

For example, Becley et al. found increased aggregation of a cysteine-linked ADC at higher temperatures and for the species with a higher DAR [166]. This trend was attributed to the drug conjugation in the hinge region, as revealed by DSC and CD data. The authors note that for the species with a higher drug loading, while conjugation does not measurably alter the secondary structure, it does reduce the thermal stability of the $\mathrm{CH} 2$ domain, with high molecular weight aggregates forming rapidly. Using SEC, Adem et al. showed that the aggregation propensity of an auristatin ADC increases with the ionic strength of the solution, especially again for the high drug load species [167]. The authors then used DSC enables to directly correlate the thermal unfolding of the protein with the loading of the drug.

Regarding the photosensitivity of ADCs, although those currently on the market are conjugated with non-photosensitive drugs, many of those under clinical trial contain photosensitive drugs such as doxorubicin or duocarmycin derivatives. Cockrell et al. reported that binding an eosin photosensitizer to trastuzumab promotes the formation of aggregates, with high-mass components being detected by SEC analysis and dynamic light scattering highlighting the formation of large soluble particles and aggregates [168]. Since aggregation 
occurred for the conjugate but not on the $\mathrm{mAb}$ control, this indicates that drug conjugation is specifically responsible for the observed aggregation.

The presence of charge variants can also affect the stability, solubility, pharmacokinetic and tissue distribution of an ADC. Boylan et al. used SEC-MS to determine the DAR and evaluate the proportion of aggregates for different charge variants of a cysteinelinked Fc fragment, the heterogeneity originating from degradation and different drug-linkers and conjugation sites [106]. For a species at a given DAR, several acidic bands are observed in the IEF data, revealing the presence of charge variants. The authors did not find any correlation between particular conjugation sites and charge variants.

In the future, native MS approaches should become more widespread for the analysis of $\mathrm{ADC}$ and $\mathrm{mAb}$ aggregates, as the on-line coupling of SEC to native MS makes the the analysis of aggregates straightforward. A few groups have already exploited HDX to study $\mathrm{mAb}$ aggregation. Zhang et al. used HDX-MS to compare the aggregation mechanism and the resulting aggregate structures of a mAb under freeze-thaw and thermal stress, showing that bevacizumab aggregation increases with the number of $\mathrm{F} / \mathrm{T}$ cycles and decreases with the protein concentration [150]. The reduced hydrogen exchange recorded for three complementarity determining regions suggests that these residues may form strong intermolecular bonds in the antibody aggregates, while the regions with enhanced HDX rates are most probably partially unfolded. An attempt was made to predict the aggregation patterns using several residue level modeling methods, but this proved unsuccessful [150]. 


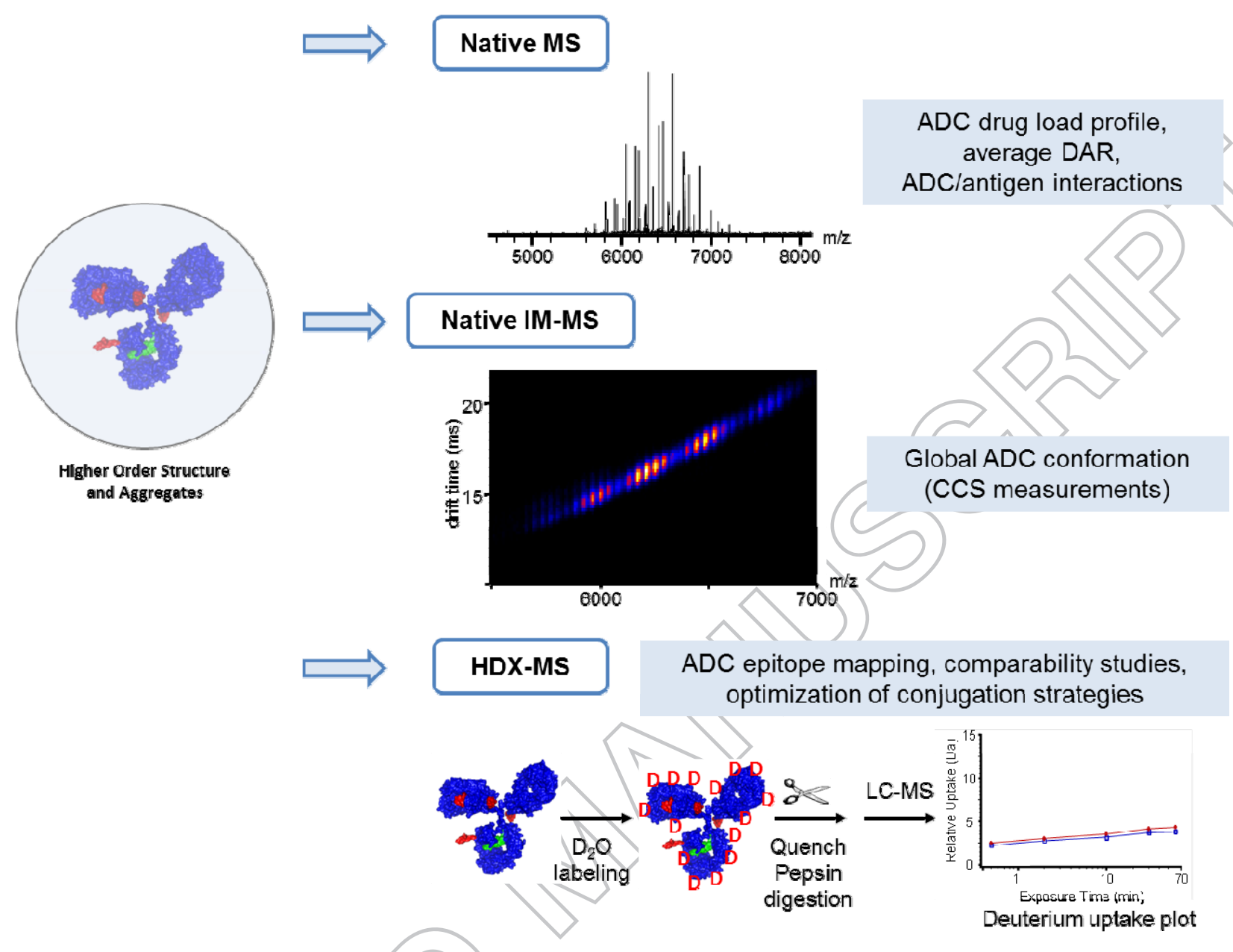

Figure 8. Mass spectrometry techniques for the analysis of higher order structure and aggregation for ADCs.

\section{Residual drug linker and related products}

As highlighted in Table 1 and already mentioned above as a potential health hazard, the amount of residual drug-linker and related products in ADC drug substance batches is a critical quality attribute. The unconjugated drug or drug-related impurities that remain in the final product are typically the result of incomplete purification down-stream of the conjugation reaction. Related forms of the unconjugated drug, such as linker-drug species or other degradation products, may also be released while the conjugate is in storage. Sensitive methods are therefore required in ADC development and production facilities to monitor and 
validate the cleaning procedures for cytotoxic products, both for the safety of operators and to demonstrate the absence of cross contamination in multi-cytotoxic production plants [169].

Wakanhar et al. have reviewed ELISA, HPLC-UV/Vis and CE-LIF (capillary electrophoresis laser induced fluorescence) methods that have been used to determine the concentrations of free drug molecules in various ADCs [44]. Chih et al. also emphasize the importance of monitoring drug-linker release with an example MS application [170]. This method was successfully used to explain an unexpected release of free drug during the stability testing of ADCs.

One of the major practical challenges of these kinds of analyses is removing the large ADC excess from the sample to allow the very small amounts of multiple small organic molecules to be quantified. This issue was solved by Fleming et al. for free DM1 dosages by directly injecting the ADC onto a single rpHPLC column without prior sample preparation [54]. The ADC flows through the column without interacting with the stationary phase, which combines a hydrophobic core with a hydrophilic outer layer. The hydrophilic layer shields the C18-like core from interactions with $\mathrm{mAbs}$ and ADCs, while the small organic molecules present in solution readily interact with the hydrophobic portion. Li et al. recently reported another elegant method based on two-dimensional LC-MS [171]. The SEC method in the $1^{\text {st }}$ dimension separated the small molecule impurities (the free drug, drug-linker, and druglinker-N-Acetylcystein adduct) from the intact ADC, and provided simultaneously information on size variants, namely monomers, dimers and aggregates. The small molecules giving rise to the peak in the $1^{\text {st }}$ dimension were isolated and further analyzed by rpHPLC in a $2^{\text {nd }}$ dimension for identification and quantitation by MS. 


\section{Bioanalysis of ADC biotransformation}

Bioanalytical methods are rapidly being developed to quantitatively monitor the transformation of ADCs in various in vitro or in vivo biological matrices such as serum/plasma and tumor tissues [172]. Indeed, a crucial property of conjugates is their stability in biofluids as the release over time of cytotoxic drugs into the bloodstream constitutes a considerable health threat [173]. This drug loss also affects the composition of the ADC, potentially altering the amount of drug delivered to the tumor site and posing another substantial safety risk because of its off-target toxicity [174]. Tumey et al. have recently published an interesting survey of the biotransformation events that have been elucidated in recent years [175], as well as one of the resulting strategies to optimize the design of next generation ADCs [115].

Traditionally, pharmacokinetic studies of $\mathrm{mAb}$ candidates are performed using ligandbinding assays (LBAs) [172,176,177], a technique also used to study the catabolites [178] and immunogenicity of ADCs [179,180]. The limitations of this approach are well known however. The specific assay reagents required for LBA are often not available early on in a program; moreover, interference can occur from endogenous proteins, antidrug antibodies and soluble target ligands [176,181]. Complementary or alternative data from liquid chromatography coupled to MS-based methods can therefore facilitate the analysis of mAbs in biological matrixes. Selected reaction monitoring (SRM)-MS combined with stable isotope dilution is thus increasingly employed in pharmacokinetic studies of recombinant proteins, notably mAbs, in highly complex matrices (serum, plasma, tumor tissues, and other body fluids) [181-187]. In addition, while LC-SRM has been used for decades to quantify small molecules, tremendous improvements in the technique over the past few years have broadened its scope to include the quantitation of peptides and proteins, including ADC catabolites [188]. Optimized LC-SRM assays offer unequalled sensitivity, high analyte 
specificity, a high multiplexing capacity and precision, and robust quantitation of analytes down to high $\mathrm{ng} / \mathrm{ml}-\mathrm{low} \mu \mathrm{g} / \mathrm{ml}$ concentrations in unfractionated plasma.

Most of the methods described above rely on enzymatic digestion of the mAb to yield at least one peptide with a unique sequence to be quantified as a surrogate for the whole mAb [189]. Multidimensional chromatography or immunoenrichment can be used to separate the $\mathrm{mAb}$ of interest from those of the endogenous matrix, thereby increasing the relative concentration of the analyte of interest [190,191]. The quantitative bioanalysis of ADCs in plasma has also be achieved by hybrid immuno-capture LC-MS/MS [192,193].

ADCs are administered as intravenous infusions, and, following in vivo processing, multiple analytes are detected in systemic circulation. According to a recent paper from the American Association of Pharmaceutical Scientists ADC Working Group [194] the most commonly observed analytes are conjugated antibodies (i.e. with a DAR of one or more), total antibodies (conjugated, partially or fully deconjugated), antibody-conjugated drugs (small molecules conjugated to an antibody), and unconjugated drug molecules. Metabolites of the drug molecule, with or without the linker, may also be detected. As discussed above, most of the ADCs currently under clinical trial exist as a heterogeneous mixture of antibody species with DARs varying from zero to eight. Each of these species has its own distinct in vivo pharmacokinetic profile, efficacy, and safety [46] and specific quantitative analytical workflows have to be developed. Pharmacokinetic profiling highlights the impact of the DAR and other perfectible parameters on the biological properties of ADCs, notably the rate of drug loss (from deconjugation and instability) and the clearance of the species with different DARs. Ultimately through, establishing the relationships between pharamacokinetic exposures and the efficacy and toxicity of the drug is the most helpful guide for the optimization and development of ADCs. This information is also mandatory for the pharmacokinetic-pharmacodynamic modeling that helps to determine the first-in human 
dosage for clinical trials [195-198]. In this context, Deslandes has recently compared the pharmacokinetics of ADCs in Phase I clinical studies [199], while toxicology issues have been discussed by Saber and Leighton from the FDA [200].

\section{Final conclusions}

The development and optimization of ADCs are increasingly reliant on the analytical and bioanalytical characterization of their main quality attributes, namely the drug load distribution, proportion of naked antibody, and DAR. These needs have recently been fulfilled by a number of cutting-edge mass spectrometry methods, with workflows optimized to be used at different levels. Electrophoretic and chromatographic methods, in association or not with MS, are still the main techniques used for the characterization of mAbs and ADC. However, recent developments in native MS mean that this technique, in combination with classical MS approaches, offers the means to complete a multi-level characterization of ADCs and other protein-based therapeutic agents.

At the top level, native MS and native IM-MS complement the data provided by HIC and rpHPLC-MS, the reference methods for the quality control of interchain cysteine- and lysine-linked ADCs, respectively.

At the middle level, the LC-MS analysis of reduced or IdeS-digested ADCs provides structural insights into the ADC subunits. The use of IdeS ensures the complete separation of the Fe/2 and Fd fragments. This facilitates the glycoprofiling of the Fc/2 fragment and the detection of any additional conjugation.

At the bottom level, high-quality ADC peptide mapping via improved (nano)HPLCMS is now available in most analytical labs. This can be used to characterize the drug-loaded peptides and to identify positional isomers at cysteine residues. Because of the hydrophobicity of the drug molecules, organic solvents are added at all stages of the bottom-up approach, including enzymatic digestion, to ensure that the drug-loaded peptides remain in solution. 
For studies of aggregation and the HOSs of ADCs, native MS and IM-MS along with HDX-MS are emerging as promising techniques to complement already established methods such as SEC.

Regarding MS-based quantitation finally, it is noteworthy that SRM-MS now offers the same sensitivity as ELISA assays, but with a superior specificity.

\section{Expert commentary \& five year view}

The state-of-the-art MS methods currently used to characterize mAbs, notably for bottomlevel analysis [201], are readily applicable for the study of ADCs. Capillary electrophoresis coupled to MS is now established as a valuable method, orthogonal to LC, for peptide mapping at the bottom [202-204] and middle [205] levels, and progress toward top level analysis is forthcoming, including under native conditions. Another emerging technique, CEUV/MALDI-MS/MS (MALDI: matrix-assisted laser desorption ionization), has recently been applied to study mAbs [206] and should prove valuable in the future.

Top-down MS and sequencing [207], middle-down MS with electron transfer dissociation fragmentation [63], and extended bottom-up proteomics with enzymes [100] are all in constant progress. An alternative middle-down avenue toward a full sequence coverage of IdeS-digested and reduced fragments of IgG may be LC-MALDI with in-source decay [207]. A common objective of all approaches based on MS/MS is to limit both the number of sample preparation steps and the use of enzymes for peptide mapping to avoid the introduction of artifacts into the data (e.g. over-deamidation from the use of trypsin at basic $\mathrm{pH})$.

Two-dimensional LC-MS should facilitate future studies of large molecules such as antibodies and ADCs, the extra resolution enabling the direct identification of different species. The first dimension can be adapted to the problem at hand via a plethora of chromatographic techniques developed for the characterization of antibodies, while rpHPLC 
is frequently used in the second dimension as it desalts the sample before its introduction into the mass spectrometer. Recent applications of this approach reported by Li et al. [171], Stoll et al. [208] and Birdsall et al. [98], have involved SEC x rpHPLC-MS, CEX x rpHPLC-MS, and HIC x rpHPLC-MS, respectively. Recent improvements made to chromatographic methods such as SEC [209], cation exchange [210], rpHPLC [211], and HIC (Fekete S et al, submitted) will no doubt also benefit the application of 2D-LC-MS for the analysis of mAbs, ADCs and related products.

Imaging mass spectrometry has successfully been used to reveal the spatial distribution of small drugs and metabolites in biological tissues [212], and has recently been demonstrated for the study of large biological molecules [213], notably to monitor mAbs in brain tumors [214]. One assumes therefore that this technique will also be applied for the characterization of ADCs.

Focusing now on the samples under study, while on the one hand, next-generation ADCs should be more homogeneous and thereby easier to characterize, their more complex formulation will pose new analytical questions. Indeed, bi-specific antibody conjugates [215], antibody-dual-conjugates [216], treatment combinations (eg. ADCs + immune check points mAbs) as well as alternatives to IgG formats [217] have already been developed.

Finally, a feasible objective for the analytical methods used to study ADCs during pharmaceutical development, is that they should be translated to the bioanalytical field, for the analysis of blood and/or tumor tissues. This would allow the quantitation of known and unknown catabolites for large and small molecules and help to optimize the next generation of ADCs. 


\section{Key issues}

- High resolution native MS provides accurate mass measurements (within $30 \mathrm{ppm}$ ) of intact ADCs, and can also yield the average DAR and drug load distribution. Native MS is furthermore unique in its ability to simultaneous detect covalent and non-covalent species in a mixture.

- Native IM-MS reveals the drug loading profile of ADCs, the CCCs of each payload species highlighting slight conformational differences.

- As an orthogonal method, LC-MS following IdeS digestion of ADCs can be used to measure the drug load distribution on light chain and Fd fragments, as well as the average DAR for both monomeric and multimeric species. In addition, the Fc fragment can be analyzed in the same run, providing a complete glyco-profile and demonstrating the presence or absence of additional conjugation.

- Because the drug molecules are hydrophobic, all enzymatic digestion steps used to prepare the sample for peptide mapping have to be adapted to keep the drug-loaded peptides soluble. When this procedure is properly optimized however, unambiguous maps are obtained by LC-MS, while the positional isomers of the cytotoxic drug can be determined by rpHPLC after digestion with IdeS and reduction.

\section{Acknowledgments}

The authors would like to thank all the members of the CIPF and LSMBO that have been involved in the characterization of ADCs: A. Boeuf, M. Excoffier, L. Maurel-Chevillet, C. Klinguer-Hamour, T. Champion. M. Dillenbourg, M. Fumex, S. Tanguy, F. Delalande, H. Diemer, J.-M. Strub, C. Carapito and C. Schaeffer.

They would also like to thank Paul Guerry from Green Grow Scientific for editing of the manuscript. 


\section{Financial and competing interests disclosure}

This work was supported by the bioclusters of the OptimAbs network (the Lyon Biopole and Alsace Biovalley) and sponsors (DGCIS, Oseo, Feder, Région Rhône-Alpes, Région Alsace, Communauté Urbaine de Strasbourg, CNRS, and the Université de Strasbourg).

We thank GIS IBiSA and Région Alsace for financial support in purchasing respectively a Synapt G2 HDMS instrument and a Exactive Plus EMR Orbitrap spectrometer.

François Debaene is an employee of Sanofi. The other authors have no other relevant affiliations or financial involvement with any organization or entity with a financial interest in or financial conflict with the subject matter or materials discussed in the manuscript. This includes employment, consultancies, honoraria, stock ownership or options, expert testimony, grants or patents received or pending, or royalties. 
Table 1. Analytical, structural and functional assays reported in quality control monographs for the characterization of ADCs. They may be used for drug substance/drug product batch release or characterization as well as for comparability, stability studies and bioanalysis). Mass spectrometry-related methods appear in bold.

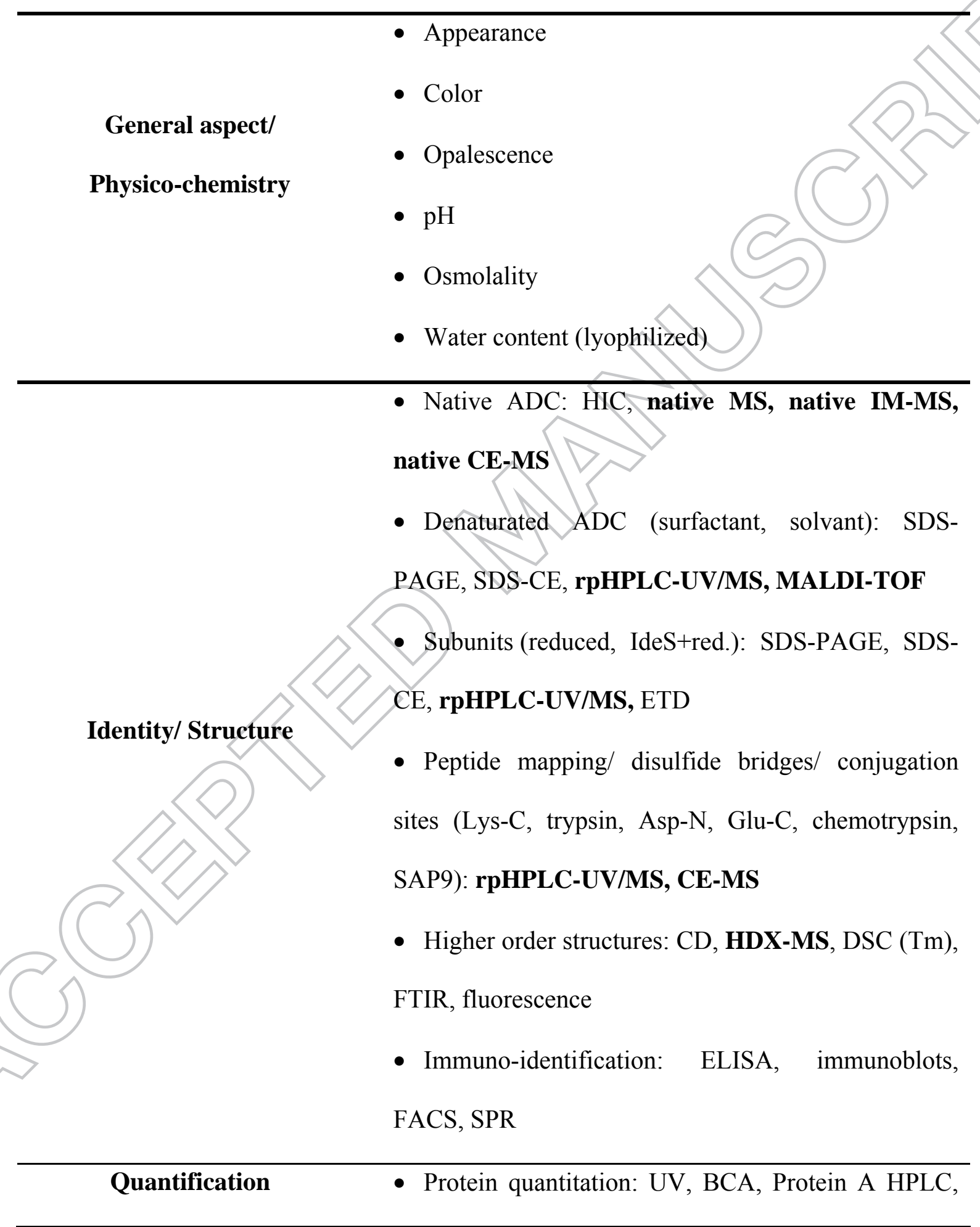




\section{ELISA}

- Extinction coefficient: calculation, amino acid analysis

- Mass variants/ Aggregates: SDS-CE, SDS-PAGE, SEC-UV/MALS/IR， LC-MS， A4F-UV/MALS/IR, DLS, SV-AUC, native MS, IM-MS

- Subvisible/visible particles: light obscuration, MFI/MCM (Flow Cell Microscopy)

- Charge variants (pI): IEF, cIEF, icIEF/iCE, CEX

- Drug loading and distribution, average DAR, positional isomers. UV, HIC, LC-UV/MS (red; IdeS/red), 2D-LC-UV/MS, native MS

\section{Purity/ Impurities}

- Free antibody: HIC, cIEF, icIEF/iCE

- Free drug(linker) and related products: LCUV/MS, 2D-LC-UV/MS, ELISA

- Free thiol groups: Ellman's reagent

- Quenching agent (N-acetyl cysteine): LC-UV/MS

- Residual solvent: LC-UV, GC

- Heavy metals: ICP-MS

- Endotoxins

- Bioburden

- Antigen binding: ELISA, FACS, SPR, native MS

Functional assays/ Potency

- Cytotoxicity (IC50): cell based assays (FACS, electroluminescence, UV-visible) 


\section{- ADCC (when applied)}

A4F, asymmetrical flow field-flow fractionation; ADCC, antibody-dependent cell-mediated cytotoxicity; BCA, bicinchoninic acid; CD, circular dichroism; CEX, cation exchange; DAR, drug-to-antibody ratio; DSC, differential scanning calorimetry; ELISA, enzyme-like immunosorbent assay; ETD, electron transfer dissociation; FACS, Fluorescence-activated cell sorting; FTIR, Fourier transform infrared; HDX, hydrogen/deuterium exchange; HIC, hydrophobic interaction chromatography; (i)CE, (imaged) capillary electrophoresis; (i)(c)IEF, (imaged) (capillary) isoelectrofocusing; ICP, inductively coupled plasma; IdeS, the immunoglobulin-degrading enzyme of Streptococcus pyogenes; IM, ion mobility; IR, infrared; MALDI, matrix-assisted laser desorption ionization; MALS, multiangle light scattering; MS, mass spectrometry; PAGE, polyacrylamide gel electrophoresis; rpHPLC, reverse-phase high-performance liquid chromatography; SDS, sodium dodecyl sulphate; SEC, size exclusion chromatography; SPR, surface plasmon resonance; SV-AUC, sedimentation velocity analytical ultracentrifugation; TOF, time of flight; UV, ultraviolet. 
References Papers of special note have been highlighted as:

. of interest

.. of considerable interest

1. Beck A, Wurch T, Bailly C, Corvaia N. Strategies and challenges for the next generation of therapeutic antibodies. Nat Rev Immunol, 10(5), 345-352 (2010).

2. Reiner Z. PCSK9 inhibitors - past, present and future. Expert Opin Drug Metab Toxicol, 1-5 (2015).

3. Reichert JM. Antibodies to watch in 2015. MAbs, 7(1), 1-8 (2015).

4. Kraeber-Bodere F, Bodet-Milin C, Rousseau C et al. Radioimmunoconjugates for the treatment of cancer. Semin Oncol, 41(5), 613-622 (2014).

5. Beck A, Haeuw JF, Wurch T, Goetsch L, Bailly C, Corvaia N The next generation of antibody-drug conjugates comes of age. Discov Med, 10(53), 329-339 (2010).

6. Madhumathi J, Devilakshmi S, Sridevi S, Verma RS. Immunotoxin therapy for hematologic malignancies: where are we heading? Drug Discov Today, (2015).

7. Danielli R, Patuzzo R, Ruffini PA et al. Armed antibodies for cancer treatment: a promising tool in a changing era. Cancer Immunol Immunother, 64(1), 113-121 (2015).

8. Mendes D, Alves C, Afonso $\mathrm{N}$ et al. The benefit of HER2-targeted therapies on overall survival of patients with metastatic HER2-positive breast cancer - a systematic review. Breast Cancer Res, 17(1), 140 (2015).

9. Beck A. Review of Antibody-Drug Conjugates, Methods in Molecular Biology series: A book edited by Laurent Ducry. MAbs, 6(1), 30-33 (2014).

10. Klinguer-Hamour C, Strop P, Shah DK, Ducry L, Xu A, Beck A. World Antibody-Drug Conjugate Summit, October 15-16, 2013, San Francisco, CA. MAbs, 6(1), 18-29 (2014).

11. Beck A, Reichert JM. Antibody-drug conjugates: present and future. MAbs, 6(1), 1517 (2014).

12. Sochaj AM, Swiderska KW Otlewski J. Current methods for the synthesis of homogeneous antibody-drug conjugates. Biotechnol Adv, 33(6 Pt 1), 775-784 (2015).

13. Beck A, Senter R, Chari R. World Antibody Drug Conjugate Summit Europe: February 21-23, 2011, Frankfurt, Germany. MAbs, 3(4), 331-337 (2011).

14. Maeda E, UrakamiK, Shimura K, Kinoshita M, Kakehi K. Charge heterogeneity of a therapeutic monoclonal antibody conjugated with a cytotoxic antitumor antibiotic, calicheamicin. Journal of Chromatography A, 1217(45), 7164-7171 (2010).

15. Labrijn AF, Buijsse AO, van den Bremer ET et al. Therapeutic IgG4 antibodies engage in Fab-arm exchange with endogenous human IgG4 in vivo. Nat Biotechnol, 27(8), 767-771 (2009).

16. Bross PF, Beitz J, Chen $G$ et al. Approval summary: gemtuzumab ozogamicin in relapsed acute myeloid leukemia. Clin Cancer Res, 7(6), 1490-1496 (2001).

17. Chari RV, Miller ML, Widdison WC. Antibody-drug conjugates: an emerging concept in cancer therapy. Angew Chem Int Ed Engl, 53(15), 3796-3827 (2014).

18. Ornes S. Antibody-drug conjugates. P Natl Acad Sci USA, 110(34), 13695-13695 (2013).

19. Flygare JA, Pillow TH, Aristoff P. Antibody-drug conjugates for the treatment of cancer. Chem Biol Drug Des, 81(1), 113-121 (2013).

20. Beck A, Lambert J, Sun M, Lin K. Fourth World Antibody-Drug Conjugate Summit: February 29-March 1, 2012, Frankfurt, Germany. MAbs, 4(6), 637-647 (2012). 
21. Doronina SO, Toki BE, Torgov MY et al. Erratum: Development of potent monoclonal antibody auristatin conjugates for cancer therapy (vol 21, pg 778, 2003). Nature Biotechnology, 21(8), 941-941 (2003).

22. Senter PD, Sievers EL. The discovery and development of brentuximab vedotin for use in relapsed Hodgkin lymphoma and systemic anaplastic large cell lymphoma. Nat Biotechnol, 30(7), 631-637 (2012).

**Adcetris ${ }^{*}:$ from bench to the FDA approval

23. Lewis Phillips GD, Li G, Dugger DL et al. Targeting HER2-positive breast cancer with trastuzumab-DM1, an antibody-cytotoxic drug conjugate. Cancer Res, 68(22), 92809290 (2008).

24. Blanc V, Bousseau A, Caron A, Carrez C, Lutz RJ, Lambert JM. SAR3419: an antiCD19-Maytansinoid Immunoconjugate for the treatment of B-cell malignancies. Clin Cancer Res, 17(20), 6448-6458 (2011).

25. Wang L, Amphlett G, Blattler WA, Lambert JM, Zhang W. Structural characterization of the maytansinoid-monoclonal antibody immunoconjugate, huN901-DM1, by mass spectrometry. Protein Sci, 14(9), 2436-2446 (2005).

26. Roberts SA, Andrews PA, Blanset $D$ et al. Considerations for the nonclinical safety evaluation of antibody drug conjugates for oncology. Regul Toxicol Rharmacol, 67(3), 382-391 (2013).

27. Gordon M, Canakci M, Li L, Zhuang J, Osborne BA, Thayumanavan S. A Field Guide to Challenges and Opportunities in Antibody-Drug Conjugates for Chemists. Bioconjug Chem, (2015).

28. McDonagh CF, Turcott E, Westendorf $L$ et al. Engineered antibody-drug conjugates with defined sites and stoichiometries of drug attachment. Protein Eng Des Sel, 19(7), 299-307 (2006).

29. Hartley JA, Hamaguchi A, Coffils M et al SG2285, a novel C2-aryl-substituted pyrrolobenzodiazepine dimer prodrug that cross-links DNA and exerts highly potent antitumor activity. Cancer Res, 70(17), 6849-6858 (2010).

30. Junutula JR, Raab H, Clark $\mathrm{S}$ et al. Site-specific conjugation of a cytotoxic drug to an antibody improves the therapeutic index. Nat Biotechnol, 26(8), 925-932 (2008).

${ }^{* *}$ Reference paper for site-specific antibody drug conjugates.

31. Kung Sutherland MS, Walter RB, Jeffrey SC et al. SGN-CD33A: a novel CD33targeting antibody-drug conjugate using a pyrrolobenzodiazepine dimer is active in models of drug-resistant AML. Blood, 122(8), 1455-1463 (2013).

32. Dennler $\mathrm{P}$, Chiotellis A, Fischer $\mathrm{E}$ et al. Transglutaminase-Based Chemo-Enzymatic Conjugation Approach Yields Homogeneous Antibody-Drug Conjugates. Bioconjugate Chem, 25(3), 569-578 (2014).

33. Farias SE, Strop $\mathrm{P}$, Delaria $\mathrm{K}$ et al. Mass spectrometric characterization of transglutaminase based site-specific antibody-drug conjugates. Bioconjug Chem, 25(2), 240-250 (2014).

34. Axup JY, Bajjuri KM, Ritland $\mathrm{M}$ et al. Synthesis of site-specific antibody-drug conjugates using unnatural amino acids. Proc Natl Acad Sci U S A, 109(40), 1610116106 (2012).

35. Smith EL, Giddens JP, lavarone AT, Godula K, Wang LX, Bertozzi CR. Chemoenzymatic Fc glycosylation via engineered aldehyde tags. Bioconjug Chem, 25(4), 788-795 (2014).

36. Kolodych S, Koniev O, Baatarkhuu $\mathrm{Z}$ et al. CBTF: new amine-to-thiol coupling reagent for preparation of antibody conjugates with increased plasma stability. Bioconjug Chem, 26(2), 197-200 (2015).

37. Koniev O, Kolodych S, Baatarkhuu Z et al. MAPN: First-in-Class Reagent for Kinetically Resolved Thiol-to-Thiol Conjugation. Bioconjug Chem, (2015).

38. Jeffrey SC, Burke PJ, Lyon RP et al. A potent anti-CD70 antibody-drug conjugate combining a dimeric pyrrolobenzodiazepine drug with site-specific conjugation technology. Bioconjug Chem, 24(7), 1256-1263 (2013). 
39. Dorywalska M, Strop P, Melton-Witt JA et al. Effect of attachment site on stability of cleavable antibody drug conjugates. Bioconjug Chem, 26(4), 650-659 (2015).

40. Strop P, Delaria K, Foletti D et al. Site-specific conjugation improves therapeutic index of antibody drug conjugates with high drug loading. Nat Biotechnol, 33(7), 694696 (2015).

${ }^{*}$ Site-specific drug conjugation technology that overcome the previously reported limitations of conventional high-loaded ADCs..

41. Alley SC, Anderson KE. Analytical and bioanalytical technologies for characterizing antibody-drug conjugates. Curr Opin Chem Biol, 17(3), 406-411 (2013).

42. Reichert JM, Beck A, Lugovskoy AA, Wurch T, Coats S, Brezski RJ. 9th annual European Antibody Congress, November 11-13, 2013, Geneva, Switzerland MAbs, 6(2), 309-326 (2014).

43. Hamilton GS. Antibody-drug conjugates for cancer therapy: The technological and regulatory challenges of developing drug-biologic hybrids. Biologicals, (2015).

44. Wakankar A, Chen Y, Gokarn Y, Jacobson FS. Analytical methods for physicochemical characterization of antibody drug conjugates. MAbs, 3(2), 161-172 (2011).

** Reference review for analytical characterization of ADCs.

45. Chen Y. Drug-to-antibody ratio (DAR) by UV/Vis spectroscopy. Methods Mol Biol, 1045, 267-273 (2013).

46. Hamblett KJ, Senter PD, Chace DF et al. Effects of drug loading on the antitumor activity of a monoclonal antibody drug conjugate. Clin Cancer Res, 10(20), 70637070 (2004).

47. Le LN, Moore JMR, Ouyang J, Chen XY, Nguyen MDH, Galush WJ. Profiling Antibody Drug Conjugate Positional Isomers: A System-of-Equations Approach. Analytical Chemistry, 84(17), 7479-7486 (2012).

* Mathematical approach using inputs from capillary electrophoresis and hydrophobic interaction chromatography to determine the positional isomer distribution within a population of ADCs

48. Ouyang J. Drug-to-antibody ratio (DAR) and drug load distribution by hydrophobic interaction chromatography and reversed phase high-performance liquid chromatography. Methods Mol Biol, 1045, 275-283 (2013).

49. Haverick M, Mengisen S, Shameem M, Ambrogelly A. Separation of mAbs molecular variants by analytical hydrophobic interaction chromatography HPLC: overview and applications. MAbs, 6(4), 852-858 (2014).

*Reference review for hydrophobic interaction chromatography of mAbs and ADCs.

50. Sun MM, Beam KS, Cerveny CG et al. Reduction-alkylation strategies for the modification of specific monoclonal antibody disulfides. Bioconjug Chem, 16(5), 12821290 (2005).

**Reference research paper for interchain cysteine-drug conjugates characterization.

51. King HD, Dubowchik GM, Mastalerz $\mathrm{H}$ et al. Monoclonal antibody conjugates of doxorubicin prepared with branched peptide linkers: inhibition of aggregation by methoxytriethyleneglycol chains. J Med Chem, 45(19), 4336-4343 (2002).

52. Hollander I, Kunz A, Hamann PR. Selection of reaction additives used in the preparation of monomeric antibody-calicheamicin conjugates. Bioconjug Chem, 19(1), 358-361 (2008).

53. Quiles S, Raisch KP, Sanford LL, Bonner JA, Safavy A. Synthesis and preliminary biological evaluation of high-drug-load paclitaxel-antibody conjugates for tumortargeted chemotherapy. J Med Chem, 53(2), 586-594 (2010).

54. Fleming MS, Zhang W, Lambert JM, Amphlett G. A reversed-phase high-performance liquid chromatography method for analysis of monoclonal antibody-maytansinoid immunoconjugates. Anal Biochem, 340(2), 272-278 (2005).

55. Beck A, Sanglier-Cianferani S, Van Dorsselaer A. Biosimilar, biobetter, and next generation antibody characterization by mass spectrometry. Anal Chem, 84(11), 4637-4646 (2012). 
56. Beck A, Wagner-Rousset E, Ayoub D, Van Dorsselaer A, Sanglier-Cianferani S. Characterization of therapeutic antibodies and related products. Anal Chem, 85(2), 715-736 (2013).

${ }^{* *}$ Comprehensive review of mAbs analytical and structural characterization.

57. Beck A, Diemer H, Ayoub D et al. Analytical characterization of biosimilar antibodies and Fc-fusion proteins. Trac-Trend Anal Chem, 48, 81-95 (2013).

58. Beck A, Debaene F, Diemer $\mathrm{H}$ et al. Cutting-edge mass spectrometry characterization of originator, biosimilar and biobetter antibodies. J Mass Spectrom, 50(2), 285-297 (2015).

59. Beck A, Cianférani S. Harness the benefits of mass spectrometry for in-depth Antibody Drug Conjugates analytical characterization. LCGC, in press (2015).

60. Fornelli L, Damoc E, Thomas PM et al. Analysis of Intact Monoclonal Antibody IgG1 by Electron Transfer Dissociation Orbitrap FTMS. Molecular \& Cellular Proteomics, 11(12), 1758-1767 (2012).

61. Tsybin YO, Fornelli L, Stoermer $\mathrm{C}$ et al. Structural analysis of intact monoclonal antibodies by electron transfer dissociation mass spectrometry. Anal Chem, 83(23), 8919-8927 (2011).

62. Mao Y, Valeja SG, Rouse JC, Hendrickson CL, Marshall AG. Top-down structural analysis of an intact monoclonal antibody by electron capture dissociation-Fourier transform ion cyclotron resonance-mass spectrometry Anal Chem, 85(9), 4239-4246 (2013).

63. Fornelli L, Ayoub D, Aizikov K, Beck A, Tsybin YO. Middle-Down Analysis of Monoclonal Antibodies with Electron Transfer Dissociation Orbitrap Fourier Transform Mass Spectrometry. Analytical Chemistry, 86(6), 3005-3012 (2014).

64. Valliere-Douglass JF, McFee WA, Salas-Solano O. Native intact mass determination of antibodies conjugated with monomethyl Auristatin $\mathrm{E}$ and $\mathrm{F}$ at interchain cysteine residues. Anal Chem, 84(6), 2843-2849 (2012).

** First reasearch paper on native MS of ADCS

65. Debaene F, Boeuf A, Wagner-Rousset E et al. Innovative Native MS Methodologies for Antibody Drug Conjugate Characterization: High Resolution Native MS and IM-MS for Average DAR and DAR Distribution Assessment. Analytical Chemistry, 86(21), 10674-10683 (2014).

** First reasearch paper on native Ion Mobility MS of ADCs

66. Schmidt C, Robinson $\mathrm{CV}$. Dynamic protein ligand interactions--insights from MS. FEBS J, 281(8), 1950-1964 (2014).

67. Pacholarz KJ, Garlish RA, Taylor RJ, Barran PE. Mass spectrometry based tools to investigate protein-ligand interactions for drug discovery. Chem Soc Rev, 41(11), 4335-4355 (2012).

68. Rajabi K, Ashcroft AE, Radford SE. Mass spectrometric methods to analyze the structural organization of macromolecular complexes. Methods, (2015).

69. Bereszczak JZ, Havlik M, Weiss VU et al. Sizing up large protein complexes by electrospray ionisation-based electrophoretic mobility and native mass spectrometry: morphology selective binding of Fabs to hepatitis B virus capsids. Anal Bioanal Chem, 406(5), 1437-1446 (2014).

70. Rosati S, Yang Y, Barendregt A, Heck AJ. Detailed mass analysis of structural heterogeneity in monoclonal antibodies using native mass spectrometry. Nat Protoc, 9(4), 967-976 (2014).

**Detailed protocol for mAbs native MS analytical characterizaion

71. Thompson NJ, Hendriks LJ, de Kruif J, Throsby M, Heck AJ. Complex mixtures of antibodies generated from a single production qualitatively and quantitatively evaluated by native Orbitrap mass spectrometry. MAbs, 6(1), 197-203 (2014).

72. Zhang H, Cui W, Gross ML. Mass spectrometry for the biophysical characterization of therapeutic monoclonal antibodies. FEBS Lett, 588(2), 308-317 (2014).

73. Thompson NJ, Rosati S, Heck AJ. Performing native mass spectrometry analysis on therapeutic antibodies. Methods, 65(1), 11-17 (2014). 
74. Debaene F, Wagner-Rousset E, Colas $\mathrm{O}$ et al. Time Resolved Native Ion-Mobility Mass Spectrometry to Monitor Dynamics of IgG4 Fab Arm Exchange and "Bispecific" Monoclonal Antibody Formation. Analytical Chemistry, 85(20), 9785-9792 (2013).

* 75. Rosati S, Rose RJ, Thompson NJ et al. Exploring an orbitrap analyzer for the characterization of intact antibodies by native mass spectrometry. Angew Chem Int Ed Engl, 51(52), 12992-12996 (2012).

76. Chen J, Yin S, Wu Y, Ouyang J. Development of a native nanoelectrospray mass spectrometry method for determination of the drug-to-antibody ratio of antibody-drug conjugates. Anal Chem, 85(3), 1699-1704 (2013).

77. Rosati S, van den Bremer ET, Schuurman J, Parren PW, Kamerling JP, Heck AJ. In depth qualitative and quantitative analysis of composite glycosylation profiles and other micro-heterogeneity on intact monoclonal antibodies by high-resolution native mass spectrometry using a modified Orbitrap. MAbs, 5(6), 917-924 (2013).

78. Marcoux J, Champion $\mathrm{T}$, Colas $\mathrm{O}$ et al. Native mass spectrometry and ion mobility characterization of trastuzumab emtansine, a lysine-linked antibody drug conjugate. Protein Science, 24(8), 1210-1223 (2015).

${ }^{* *}$ First research paper of Kadcyla ${ }^{\circledR}$ Native Ion Mobility MS characterization

79. Hengel SM, Sanderson R, Valliere-Douglass J, Nicholas N, Leiske C, Alley SC. Measurement of in vivo drug load distribution of cysteine-linked antibody-drug conjugates using microscale liquid chromatography mass spectrometry. Anal Chem, 86(7), 3420-3425 (2014).

**First demonstration that native MS characterization of ADCs may be used as a potent bioanalysis method.

80. Smith LM, Kelleher NL. Proteoform: a single term describing protein complexity. Nat Methods, 10(3), 186-187 (2013).

81. Tran JC, Zamdborg L, Ahlf DR et al. Mapping intact protein isoforms in discovery mode using top-down proteomics. Nature, 480(7376), 254-258 (2011).

82. Zhang Z, Shah B. Characterization of variable regions of monoclonal antibodies by top-down mass spectrometry. Anal Chem, 79(15), 5723-5729 (2007).

83. Bondarenko PV, Second TP, Zabrouskov V, Makarov AA, Zhang Z. Mass measurement and top-down HRLC/MS analysis of intact monoclonal antibodies on a hybrid linear quadrupole ion trap-Orbitrap mass spectrometer. J Am Soc Mass Spectrom, 20(8), 1415-1424 (2009).

84. Ren D, Pipes GD, Hambly D, Bondarenko PV, Treuheit MJ, Gadgil HS. Top-down Nterminal sequencing of Immunoglobulin subunits with electrospray ionization time of flight mass spectrometry. Anal Biochem, 384(1), $42-48$ (2009).

85. Lermyte F, Sobott F. Electron transfer dissociation provides higher-order structural information of native and partially unfolded protein complexes. Proteomics, 15(16), 2813-2822 (2015).

** 86. Dyachenko A, Wang G, Belov $M$ et al. Tandem Native Mass-Spectrometry on Antibody-Drug Conjugates and Submillion Da Antibody-Antigen Protein Assemblies on an Orbitrap EMR Equipped with a High-Mass Quadrupole Mass Selector. Anal Chem, 87(12), 6095-6102 (2015).

87. Beck A, Bussat MC, Zorn N et al. Characterization by liquid chromatography combined with mass spectrometry of monoclonal anti-IGF-1 receptor antibodies produced in CHO and NSO cells. J Chromatogr B Analyt Technol Biomed Life Sci, 819(2), 203-218 (2005).

* 88. Wagner-Rousset E, Janin-Bussat MC, Colas O et al. Antibody-drug conjugate model fast characterization by LC-MS following IdeS proteolytic digestion. MAbs, 6(1), 273285 (2014).

*First research report showing that IdeS may be used for ADCs subunit characterization by MS.

89. Xu W, Peng Y, Wang F, Paporello B, Richardson D, Liu H. Method to convert Nterminal glutamine to pyroglutamate for characterization of recombinant monoclonal antibodies. Anal Biochem, 436(1), 10-12 (2013). 
90. Basa L. Drug-to-antibody ratio (DAR) and drug load distribution by LC-ESI-MS. Methods Mol Biol, 1045, 285-293 (2013).

91. Lyon RP, Meyer DL, Setter JR, Senter PD. Conjugation of Anticancer Drugs through Endogenous Monoclonal Antibody Cysteine Residues. Method Enzymol, 502, 123138 (2012).

** 92. Janin-Bussat MC, Dillenbourg M, Corvaia N, Beck A, Klinguer-Hamour C. Characterization of antibody drug conjugate positional isomers at cysteine residues by peptide mapping LC-MS analysis. J Chromatogr B Analyt Technol Biomed Life Sci, 981-982, 9-13 (2015).

${ }^{*}$ First research report on Adcetris $\circledR$ peptide mapping.

93. Yan B, Valliere-Douglass J, Brady L et al. Analysis of post-translational modifications in recombinant monoclonal antibody $\operatorname{lgG} 1$ by reversed-phase liquid chromatography/mass spectrometry. J Chromatogr A, 1164(1-2), 153-161 (2007).

94. Gadgil HS, Bondarenko PV, Pipes G et al. The LC/MS analysis of glycation of /gG molecules in sucrose containing formulations. J Pharm Sci, 96(10), 2607-2621 (2007).

95. Gadgil HS, Bondarenko PV, Pipes GD et al. Identification of cysteinylation of a free cysteine in the Fab region of a recombinant monoclonal IgG1 antibody using Lys-C limited proteolysis coupled with LC/MS analysis. Anal Blochem, 355(2), 165-174 (2006).

96. Chevreux G, Tilly N, Bihoreau N. Fast analysis of recombinant monoclonal antibodies using IdeS proteolytic digestion and electrospray mass spectrometry. Anal Biochem, 415(2), 212-214 (2011).

97. Firth D, Bell L, Squires M, Estdale S, McKee C. A rapid approach for characterization of thiol-conjugated antibody-drug conjugates and calculation of drug-antibody ratio by liquid chromatography mass spectrometry. Anal Biochem, 485, 34-42 (2015).

98. Birdsall RE, Shion H, Kotch FW, Xu A, Porter TJ, Chen W. A rapid on-line method for mass spectrometric confirmation of a cysteine conjugated antibody-drug-conjugate structure using multidimensional chromatography. mAbs, in press (2015).

${ }^{* *}$ First 2D-LC characterization of cysteine ADCs positional isomers.

99. Wiggins B, Liu-Shin L, Yamaguchi H, Ratnaswamy G. Characterization of cysteinelinked conjugation profiles of immunoglobulin g1 and immunoglobulin g2 antibodydrug conjugates. J Pharm Sci, 104(4), 1362-1372 (2015).

100. Srzentic K, Fornelli $L$, Laskay UA et al. Advantages of extended bottom-up proteomics using Sap9 for analysis of monoclonal antibodies. Anal Chem, 86(19), 9945-9953 (2014).

101. Lazar AC, Wang LT, Blattler WA, Amphlett G, Lambert JM, Zhang W. Analysis of the composition of immunoconjugates using size-exclusion chromatography coupled to mass spectrometry. Rapid Commun Mass Sp, 19(13), 1806-1814 (2005).

**Key paper for the MS characterization of Antibody Maytansinoid Conjugates.

102. Lin J, Lazar AC. Determination of charge heterogeneity and level of unconjugated antibody by imaged cIEF. Methods Mol Biol, 1045, 295-302 (2013).

103. Kim MT, Chen Y, Marhoul J, Jacobson F. Statistical modeling of the drug load distribution on trastuzumab emtansine (Kadcyla), a lysine-linked antibody drug conjugate. Bioconjug Chem, 25(7), 1223-1232 (2014).

104. Lu SX, Takach EJ, Solomon M, Zhu Q, Law SJ, Hsieh FY. Mass spectral analyses of labile DOTA-NHS and heterogeneity determination of DOTA or DM1 conjugated antiPSMA antibody for prostate cancer therapy. J Pharm Sci, 94(4), 788-797 (2005).

105. Wang L, Amphlett G, Lambert JM, Blattler W, Zhang W. Structural characterization of a recombinant monoclonal antibody by electrospray time-of-flight mass spectrometry. Pharm Res, 22(8), 1338-1349 (2005).

106. Boylan NJ, Zhou W, Proos RJ, Tolbert TJ, Wolfe JL, Laurence JS. Conjugation Site Heterogeneity Causes Variable Electrostatic Properties in Fc Conjugates. Bioconjugate Chem, 24(6), 1008-1016 (2013). 
107. Huang RY, Deyanova EG, Passmore D et al. Utility of Ion Mobility Mass Spectrometry for Drug-to-Antibody Ratio Measurements in Antibody-Drug Conjugates. J Am Soc Mass Spectrom, (2015).

108. Gautier V, Boumeester AJ, Lossl P, Heck AJ. Lysine conjugation properties in human IgGs studied by integrating high-resolution native mass spectrometry and bottom-up proteomics. Proteomics, 15(16), 2756-2765 (2015).

109. Panowksi S, Bhakta S, Raab H, Polakis P, Junutula JR. Site-specific antibody drug conjugates for cancer therapy. MAbs, 6(1), 34-45 (2014).

110. Agarwal P, Bertozzi CR. Site-specific antibody-drug conjugates: the nexus of bioorthogonal chemistry, protein engineering, and drug development. Bioconjug Chem, 26(2), 176-192 (2015).

111. Dennler P, Fischer E, Schibli R. Antibody conjugates: from heterogeneous populations to defined reagents. Antibodies, 4, 197-224 (2015).

112. Shen $\mathrm{BQ}, \mathrm{Xu} \mathrm{K}$, Liu L et al. Conjugation site modulates the in vivo stability and therapeutic activity of antibody-drug conjugates. Nat Biotechnol 30(2), 184-189 (2012).

**Key paper to undestand retro-Michael deconjugation of ADCs in vivo.

113. Alley SC, Benjamin DR, Jeffrey SC et al. Contribution of linker stability to the activities of anticancer immunoconjugates. Bioconjugate Chem, 19(3), 759-765 (2008).

114. Lyon RP, Setter JR, Bovee TD et al. Self-hydrolyzing maleimides improve the stability and pharmacological properties of antibody-drug conjugates. Nat Biotechnol, 32(10), 1059-1062 (2014).

**Technology that improved ADC antitumor activity and reduced neutropenia

115. Tumey LN, Charati M, He T et al. Mild method for succinimide hydrolysis on ADCs: impact on ADC potency, stability, exposure, and efficacy. Bioconjug Chem, 25(10), 1871-1880 (2014).

116. Fontaine SD, Reid R, Robinson L, Ashley GW, Santi DV. Long-term stabilization of maleimide-thiol conjugates. Bioconjug Chem, 26(1), 145-152 (2015).

117. Okeley NM, Toki BE, Zhang XQ et al. Metabolic Engineering of Monoclonal Antibody Carbohydrates for Antibody-Drug Conjugation. Bioconjugate Chem, 24(10), 16501655 (2013).

118. van Geel R, Wijdeven MA, Heesbeen $\mathrm{R}$ et al. Chemoenzymatic Conjugation of Toxic Payloads to the Globally Conserved N-Glycan of Native mAbs Provides Homogeneous and Highly Efficacious Antibody-Drug Conjugates. Bioconjug Chem, (2015).

119. Qasba PK. Glycans of Antibodies as a Specific Site for Drug Conjugation Using Glycosyltransferases. Bioconjug Chem, (2015).

120. Janin-Bussat MC, Tonini L, Huillet $C$ et al. Cetuximab Fab and Fc N-glycan fast characterization using IdeS digestion and liquid chromatography coupled to electrospray ionization mass spectrometry. Methods Mol Biol, 988, 93-113 (2013).

121. Hallam TJ, Smider VV. Unnatural amino acids in novel antibody conjugates. Future Med Chem, 6(11), 1309-1324 (2014).

122. Perez HL, Cardarelli PM, Deshpande S et al. Antibody-drug conjugates: current status and future directions. Drug Discov Today, 19(7), 869-881 (2014).

123. Joubert MK, Hokom M, Eakin C et al. Highly aggregated antibody therapeutics can enhance the in vitro innate and late-stage T-cell immune responses. $\mathrm{J}$ Biol Chem, 287(30), 25266-25279 (2012).

124. Rosenberg AS. Effects of protein aggregates: an immunologic perspective. AAPS J, 8(3), E501-507 (2006).

125. Joubert MK, Luo Q, Nashed-Samuel Y, Wypych J, Narhi LO. Classification and characterization of therapeutic antibody aggregates. J Biol Chem, 286(28), 2511825133 (2011).

126. van Beers MM, Bardor M. Minimizing immunogenicity of biopharmaceuticals by controlling critical quality attributes of proteins. Biotechnol J, 7(12), 1473-1484 (2012). 
127. Valliere-Douglass JF, Lewis P, Salas-Solano O, Jiang S. Solid-state mAbs and ADCs subjected to heat-stress stability conditions can be covalently modified with buffer and excipient molecules. J Pharm Sci, 104(2), 652-665 (2015).

*Demonstration that freeze-dried mAbs and ADCs can be covalently modified with buffer and excipient molecules on the side chains of Glu, Asp, Thr, and Ser amino acids when subjected to temperature stress.

128. Poppe L, Jordan JB, Rogers G, Schnier PD. On the Analytical Superiority of 1D NMR for Fingerprinting the Higher Order Structure of Protein Therapeutics Compared to Multidimensional NMR Methods. Anal Chem, 87(11), 5539-5545 (2015).

129. Rayner LE, Hui GK, Gor J, Heenan RK, Dalby PA, Perkins SJ. The solution structures of two human IgG1 antibodies show conformational stability and accommodate their C1q and FcgammaR ligands. J Biol Chem, 290(13), 8420-8438 (2015).

130. Kukrer B, Filipe $\mathrm{V}$, van Duijn $\mathrm{E}$ et al. Mass spectrometric analysis of intact human monoclonal antibody aggregates fractionated by size-exclusion chromatography. Pharm Res, 27(10), 2197-2204 (2010).

131. Atmanene C, Laux A, Glattard E et al. Characterization of human and bovine phosphatidylethanolamine-binding protein (PEBP/RKIP) interactions with morphine and morphine-glucuronides determined by noncovalent mass spectrometry. Med Sci Monit, 15(7), BR178-187 (2009).

132. Haberger M, Heidenreich AK, Schlothauer T et al. Functional assessment of antibody oxidation by native mass spectrometry. MAbs, 1-10 (2015).

133. Hall Z, Politis A, Bush MF, Smith LJ, Robinson CV. Charge-state dependent compaction and dissociation of protein complexes: insights from ion mobility and molecular dynamics. J Am Chem Soc, 134(7), 3429-3438 (2012).

134. Scarff CA, Patel VJ, Thalassinos K, Scrivens JH. Probing hemoglobin structure by means of traveling-wave ion mobility mass spectrometry. J Am Soc Mass Spectrom, 20(4), 625-631 (2009).

135. Bowers MT, Kemper PR, Vonhelden G, Vankoppen PAM. Gas-Phase Ion Chromatography - Transition-Metal State Selection and Carbon Cluster Formation. Science, 260(5113), 1446-1451 (1993).

136. Ruotolo BT, Giles K, Campuzano I, Sandercock AM, Bateman RH, Robinson CV. Evidence for macromolecular protein rings in the absence of bulk water. Science, 310(5754), 1658-1661 (2005).

* 137. Bagal D, Valliere-Douglass JF, Balland A, Schnier PD. Resolving disulfide structural isoforms of IgG2 monoclonal antibodies by ion mobility mass spectrometry. Anal Chem, 82(16), 6751-6755 (2010).

138. Pacholarz KJ, Porrini M, Garlish RA et al. Dynamics of intact immunoglobulin G explored by drift-tube ion-mobility mass spectrometry and molecular modeling. Angew Chem Int Ed Engl, 53(30), 7765-7769 (2014).

139. Jones $L M$, Zhang $\mathrm{H}$, Cui $\mathrm{W}$ et al. Complementary MS methods assist conformational characterization of antibodies with altered S-S bonding networks. J Am Soc Mass Spectrom, 24(6), 835-845 (2013).

140. Pritchard C, Groves KJ, Biesenbruch S et al. Quantification of human growth hormone in serum with a labeled protein as an internal standard: essential considerations. Anal Chem, 86(13), 6525-6532 (2014).

* 141. Houde D, Berkowitz SA, Engen JR. The utility of hydrogen/deuterium exchange mass spectrometry in biopharmaceutical comparability studies. J Pharm Sci, 100(6), 20712086 (2011).

142. Kaltashov IA, Bobst CE, Abzalimov RR, Berkowitz SA, Houde D. Conformation and dynamics of biopharmaceuticals: transition of mass spectrometry-based tools from academe to industry. J Am Soc Mass Spectrom, 21(3), 323-337 (2010).

143. Guan X, Noble KA, Tao $Y$ et al. Epitope mapping of $7 \mathrm{~S}$ cashew antigen in complex with antibody by solution-phase H/D exchange monitored by FT-ICR mass spectrometry. J Mass Spectrom, 50(6), 812-819 (2015). 
144. Faleri A, Santini L, Brier S et al. Two cross-reactive monoclonal antibodies recognize overlapping epitopes on Neisseria meningitidis factor $\mathrm{H}$ binding protein but have different functional properties. FASEB J, 28(4), 1644-1653 (2014).

145. Sevy AM, Healey JF, Deng W, Spiegel PC, Meeks SL, Li R. Epitope mapping of inhibitory antibodies targeting the C2 domain of coagulation factor VIII by hydrogendeuterium exchange mass spectrometry. J Thromb Haemost, 11(12), 2128-2136 (2013).

146. Obungu VH, Gelfanova V, Huang L. Epitope mapping of antibodies by mass spectroscopy: a case study. Methods Mol Biol, 988, 291-302 (2013).

147. Pandit D, Tuske SJ, Coales SJ et al. Mapping of discontinuous conformational epitopes by amide hydrogen/deuterium exchange mass spectrometry and computational docking. J Mol Recognit, 25(3), 114-124 (2012).

148. Jensen PF, Larraillet V, Schlothauer $T$, Kettenberger $H$, Hilger M, Rand KD. Investigating the interaction between the neonatal $\mathrm{Fc}$ receptor and monoclonal antibody variants by hydrogen/deuterium exchange mass spectrometry. Mol Cell Proteomics, 14(1), 148-161 (2015).

149. Zhang A, Qi W, Singh SK, Fernandez EJ. A new approach to explore the impact of freeze-thaw cycling on protein structure: hydrogen/deuterium exchange mass spectrometry (HX-MS). Pharm Res, 28(5), 1179-1193 (2011).

150. Zhang A, Singh SK, Shirts MR, Kumar S, Fernandez EJ. Distinct aggregation mechanisms of monoclonal antibody under thermal and freeze-thaw stresses revealed by hydrogen exchange. Pharm Res, 29(1), 236-250 (2012).

** 151. lacob RE, Bou-Assaf GM, Makowski L, Engen JR, Berkowitz SA, Houde D. Investigating monoclonal antibody aggregation using a combination of H/DX-MS and other biophysical measurements. J Pharm Sci, 102(12), 4315-4329 (2013).

152. Majumdar R, Middaugh CR, Weis DD, Volkin DB. Hydrogen-deuterium exchange mass spectrometry as an emerging analytical tool for stabilization and formulation development of therapeutic monoclonal antibodies. J Pharm Sci, 104(2), 327-345 (2015).

153. Arora J, Hickey JM, Majumdar R et al. Hydrogen exchange mass spectrometry reveals protein interfaces and distant dynamic coupling effects during the reversible self-association of an IgG1 monoclonal antibody. MAbs, 7(3), 525-539 (2015).

154. Houde D, Peng Y, Berkowitz SA, Engen JR. Post-translational modifications differentially affect IgG1 conformation and receptor binding. Mol Cell Proteomics, 9(8), 1716-1728 (2010).

155. Burkitt W, Domann P, O'Connor G. Conformational changes in oxidatively stressed monoclonal antibodies studied by hydrogen exchange mass spectrometry. Protein Sci, 19(4), 826-835 (2010).

156. Zhang $\mathrm{A}$, Hu $\mathrm{P}$, MacGregor $\mathrm{P}$ et al. Understanding the conformational impact of chemical modifications on monoclonal antibodies with diverse sequence variation using hydrogen/deuterium exchange mass spectrometry and structural modeling. Anal Chem, 86(7), 3468-3475 (2014).

157. Tsuchida D, Yamazaki K, Akashi S. Characterization of stress-exposed granulocyte colony stimulating factor using ELISA and hydrogen/deuterium exchange mass spectrometry. J Am Soc Mass Spectrom, 25(10), 1747-1754 (2014).

** 158. Engen JR, Wales TE. Analytical Aspects of Hydrogen Exchange Mass Spectrometry. Annu Rev Anal Chem (Palo Alto Calif), 8, 127-148 (2015).

159. Ionescu RM, Vlasak J, Price C, Kirchmeier M. Contribution of variable domains to the stability of humanized IgG1 monoclonal antibodies. J Pharm Sci, 97(4), 1414-1426 (2008).

160. Wakankar AA, Feeney MB, Rivera J et al. Physicochemical stability of the antibodydrug conjugate Trastuzumab-DM1: changes due to modification and conjugation processes. Bioconjug Chem, 21(9), 1588-1595 (2010). 
161. Guo J, Kumar S, Prashad A, Starkey J, Singh SK. Assessment of physical stability of an antibody drug conjugate by higher order structure analysis: impact of thiolmaleimide chemistry. Pharm Res, 31(7), 1710-1723 (2014).

* 162. Pan LY, Salas-Solano O, Valliere-Douglass JF. Conformation and dynamics of interchain cysteine-linked antibody-drug conjugates as revealed by hydrogen/deuterium exchange mass spectrometry. Anal Chem, 86(5), 2657-2664 (2014).

163. Valliere-Douglass JF, Hengel SM, Pan LY. Approaches to Interchain Cysteine-Linked ADC Characterization by Mass Spectrometry. Mol Pharm, 12(6), 1774-1783 (2015).

*Key review for interchain Cysteine-linked ADC Characterization.

164. Pan J, Zhang S, Chou A, Hardie DB, Borchers CH. Fast Comparative Structural Characterization of Intact Therapeutic Antibodies Using Hydrogen-Deuterium Exchange and Electron Transfer Dissociation. Anal Chem, 87(12), 5884-5890 (2015).

165. Acchione $\mathrm{M}$, Kwon $\mathrm{H}$, Jochheim $\mathrm{CM}$, Atkins WM. Impact of linker and conjugation chemistry on antigen binding, Fc receptor binding and thermal stability of model antibody-drug conjugates. MAbs, 4(3), 362-372 (2012).

166. Beckley NS, Lazzareschi KP, Chih HW, Sharma VK, Flores HL. Investigation into temperature-induced aggregation of an antibody drug conjugate. Bioconjug Chem, 24(10), 1674-1683 (2013).

167. Adem YT, Schwarz KA, Duenas E, Patapoff TW, Galush WJ, Esue O. Auristatin Antibody Drug Conjugate Physical Instability and the Role of Drug Payload. Bioconjugate Chem, 25(4), 656-664 (2014).

168. Cockrell GM, Wolfe MS, Wolfe JL, Schoneich C. Photoinduced aggregation of a model antibody-drug conjugate. Mol Pharm, 12(6), 1784-1797 (2015).

169. Singh SK, Luisi DL, Pak RH. Antibody-Drug Conjugates: Design, Formulation and Physicochemical Stability. Pharm Res, (2015).

170. Chih HW, Gikanga B, Yang Y, Zhang B. Identification of Amino Acid Residues Responsible for the Release of Free Drug from an Antibody-Drug Conjugate Utilizing Lysine-Succinimidyl Ester Chemistry. J Pharm Sci-Us, 100(7), 2518-2525 (2011).

171. Li Y, Gu C, Gruenhagen J et al. A size exclusion-reversed phase two dimensionalliquid chromatography methodology for stability and small molecule related species in antibody drug conjugates. J Chromatogr A, 1393, 81-88 (2015).

172. Gorovits B. Bioanalysis of antibody-drug conjugates. Bioanalysis, 7(13), 1559-1560 (2015).

** Reference review for ADCs biøanalysis.

173. Zhou Q, Kim J. Advances in the Development of Site-Specific Antibody-Drug Conjugation. Anticancer Agents Med Chem, 15(7), 828-836 (2015).

174. Rao C Rangan VS, Deshpande S. Challenges in antibody-drug conjugate discovery: a bioconjugation and analytical perspective. Bioanalysis, 7(13), 1561-1564 (2015).

175. Tumey LN, Rago B, Han X. In vivo biotransformations of antibody-drug conjugates. Bioanalysis, 7(13), 1649-1664 (2015).

176. Kumar S, King LE, Clark TH, Gorovits B. Antibody-drug conjugates nonclinical support: from early to late nonclinical bioanalysis using ligand-binding assays. Bioanalysis, 7(13), 1605-1617 (2015).

177. Kelley M, Stevenson L, Golob M et al. Workshop Report: AAPS Workshop on Method Development, Validation, and Troubleshooting of Ligand-Binding Assays in the Regulated Environment. AAPS J, 17(4), 1019-1024 (2015).

178. Salomon PL, Singh R. Sensitive ELISA Method for the Measurement of Catabolites of Antibody-Drug Conjugates (ADCs) in Target Cancer Cells. Mol Pharm, 12(6), 17521761 (2015).

179. Carrasco-Triguero M. Insights on the immunogenicity of antibody-drug conjugates. Bioanalysis, 7(13), 1565-1568 (2015).

180. Carrasco-Triguero M, Yi JH, Dere R et al. Immunogenicity assays for antibody-drug conjugates: case study with ado-trastuzumab emtansine. Bioanalysis, 5(9), 10071023 (2013). 
181. Ezan E, Bitsch F. Critical comparison of MS and immunoassays for the bioanalysis of therapeutic antibodies. Bioanalysis, 1(8), 1375-1388 (2009).

${ }^{* *}$ Reference review for the complementarity of MS and ILA method of the bioanalysis of mAbs.

182. Dubois M, Becher F, Herbet A, Ezan E. Immuno-mass spectrometry assay of EPIHNE4, a recombinant protein inhibitor of human elastase. Rapid Commun Mass Spectrom, 21(3), 352-358 (2007).

183. Hagman C, Ricke D, Ewert S, Bek S, Falchetto R, Bitsch F. Absolute quantification of monoclonal antibodies in biofluids by liquid chromatography-tandem mass spectrometry. Anal Chem, 80(4), 1290-1296 (2008).

184. Heudi O, Barteau S, Zimmer D et al. Towards absolute quantification of therapeutic monoclonal antibody in serum by LC-MS/MS using isotope-labeled antibody standard and protein cleavage isotope dilution mass spectrometry. Anal Chem, 80(11), 42004207 (2008).

${ }^{* *}$ Reference research paper for quantification by LC-MS/MS mAbs in complexe biological matrixes.

185. Lesur A, Varesio E, Hopfgartner G. Accelerated tryptic digestion for the analysis of biopharmaceutical monoclonal antibodies in plasma by liquid chromatography with tandem mass spectrometric detection. J Chromatogr A, 1217(1), 57-64 (2010).

186. Mesmin C, Fenaille F, Ezan E, Becher F. MS-based approaches for studying the pharmacokinetics of protein drugs. Bioanalysis, 3(5), 477-480 (2011).

187. Becher F, Dubois M, Fenaille F, Ezan E. Mass spectrometry protocol for the absolute quantification of a monoclonal antibody in serum with immunopurification. Methods Mol Biol, 988, 345-352 (2013).

188. Saad OM, Shen BQ, Xu K, Khojasteh SC, Girish S, Kaur S. Bioanalytical approaches for characterizing catabolism of antibody-drug conjugates. Bioanalysis, 7(13), 15831604 (2015).

**Key review for ADCs catabolism.

189. Lebert D, Picard G, Beau-Larvor $\mathrm{C}$ et al. Absolute and multiplex quantification of antibodies in serum using PSAQ (TM) standards and LC-MS/MS. Bioanalysis, 7(10), 1237-1251 (2015).

*Demontration of the usefulness of PSAQ for multiplex quanntification of mAbs.

190. Ezan E, Dubois M, Becher F-Bioanalysis of recombinant proteins and antibodies by mass spectrometry. Analyst, 134(5), 825-834 (2009).

191. Liu H, Manuiloy AV, Chumsae C, Babineau ML, Tarcsa E. Quantitation of a recombinant monoclonal antibody in monkey serum by liquid chromatography-mass spectrometry, Anal Biochem, 414(1), 147-153 (2011).

192. Xu K, Liu L, Saad OM et al. Characterization of intact antibody-drug conjugates from plasma/serum in vivo by affinity capture capillary liquid chromatography-mass spectrometry. Anal Biochem, 412(1), 56-66 (2011).

193. Liu A. Pasquali M. Acidified acetonitrile and methanol extractions for quantitative analysis of acylcarnitines in plasma by stable isotope dilution tandem mass spectrometry. J Chromatogr B Analyt Technol Biomed Life Sci, 827(2), 193-198 (2005).

194. Gorovits B, Alley SC, Bilic S et al. Bioanalysis of antibody-drug conjugates: American Association of Pharmaceutical Scientists Antibody-Drug Conjugate Working Group position paper. Bioanalysis, 5(9), 997-1006 (2013).

195. Lu D, Jin JY, Girish S et al. Semi-mechanistic Multiple-Analyte Pharmacokinetic Model for an Antibody-Drug-Conjugate in Cynomolgus Monkeys. Pharm Res, 32(6), 1907-1919 (2015).

196. Xu K, Liu L, Dere R et al. Characterization of the drug-to-antibody ratio distribution for antibody-drug conjugates in plasma/serum. Bioanalysis, 5(9), 1057-1071 (2013).

**Key paper to undestand ADCs deconjugation in vivo. 
197. Khot A, Sharma S, Shah DK. Integration of bioanalytical measurements using PK-PD modeling and simulation: implications for antibody-drug conjugate development. Bioanalysis, 7(13), 1633-1648 (2015).

198. Wang H, Rangan VS, Sung MC et al. Pharmacokinetic characterization of BMS936561, an anti-CD70 antibody-drug conjugate, in preclinical animal species and prediction of its pharmacokinetics in humans. Biopharm Drug Dispos, (2015).

199. Deslandes A. Comparative clinical pharmacokinetics of antibody-drug conjugates in first-in-human Phase 1 studies. MAbs, 6(4), 859-870 (2014).

** Reference translational review for ADCs moving from the preclinic to $\mathrm{FIH}$.

200. Saber H, Leighton JK. An FDA oncology analysis of antibody-drug conjugates. Regul Toxicol Pharmacol, 71(3), 444-452 (2015).

** Reference survey of ADCs toxicity.

201. Ayoub D, Bertaccini D, Diemer $\mathrm{H}$ et al. Characterization of the N-terminal heterogeneities of monoclonal antibodies using in-gel charge derivatization of alphaamines and LC-MS/MS. Anal Chem, 87(7), 3784-3790 (2015).

202. Gahoual R, Burr A, Busnel JM et al. Rapid and multi-level characterization of trastuzumab using sheathless capillary electrophoresis-tandem mass spectrometry. MAbs, 5(3), 479-490 (2013).

**Reference research paper for the characterization of mAbs by CESI-MS.

203. Gahoual R, Biacchi M, Chicher J et al. Monoclonal antibodies biosimilarity assessment using transient isotachophoresis capillary zone electrophoresis-tandem mass spectrometry. MAbs, 6(6), 1464-1473 (2014).

204. Gahoual R, Busnel JM, Beck A, Francois YN, Leize-Wagner E. Full antibody primary structure and microvariant characterization in a single injection using transient isotachophoresis and sheathless capillary electrophoresis-tandem mass spectrometry. Anal Chem, 86(18), 9074-9081 (2014).

205. Biacchi M, Gahoual R, Said N, Beck A, Leize-Wagner E, Francois YN. Glycoform Separation and Characterization of Cetuximab Variants by Middle-up Off-Line Capillary Zone Electrophoresis-UV/Electrospray Ionization-MS. Anal Chem, 87(12), 6240-6250 (2015).

206. Biacchi M, Bhajun R, Said N, Beck A, Francois YN, Leize-Wagner E. Analysis of monoclonal antibody by a novel CE-UV/MALDI-MS interface. Electrophoresis, 35(20), 2986-2995 (2014).

207. Ayoub D, Jabs W, Resemann A et al. Correct primary structure assessment and extensive glyco-profiling of cetuximab by a combination of intact, middle-up, middledown and bottom-up ESI and MALDI mass spectrometry techniques. MAbs, 5(5), 699-710 (2013).

208. Stoll DR, Harmes DC, Danforth $\mathrm{J}$ et al. Direct Identification of Rituximab Main Isoforms and Subunit Analysis by Online Selective Comprehensive Two-Dimensional Liquid Chromatography-Mass Spectrometry. Anal Chem, 87(16), 8307-8315 (2015).

209. Fekete S, Beck A, Veuthey JL, Guillarme D. Theory and practice of size exclusion chromatography for the analysis of protein aggregates. J Pharm Biomed Anal, 101, 161-173 (2014).

${ }^{*}$ Comprehensive review of the use of SEC for mAbs characterization.

210. Fekete S, Beck A, Fekete J, Guillarme D. Method development for the separation of monoclonal antibody charge variants in cation exchange chromatography, Part I: salt gradient approach. J Pharm Biomed Anal, 102, 33-44 (2015).

211. Fekete S, Beck A, Wagner E, Vuignier K, Guillarme D. Adsorption and recovery issues of recombinant monoclonal antibodies in reversed-phase liquid chromatography. J Sep Sci, 38(1), 1-8 (2015).

212. Zhao YS, Li C. Mass Spectrometry Imaging: Applications in Drug Distribution Studies. Curr Drug Metab, (2015).

213. Neubert $P$, Walch $A$. Current frontiers in clinical research application of MALDI imaging mass spectrometry. Expert Rev Proteomics, 10(3), 259-273 (2013). 
214. Ait-Belkacem R, Berenguer C, Villard C et al. Monitoring therapeutic monoclonal antibodies in brain tumor. MAbs, 6(6), 1385-1393 (2014).

${ }^{\star *}$ First demontrastion that MALDI Imaging can be applied to mAbs.

215. Kontermann RE, Brinkmann U. Bispecific antibodies. Drug Discov Today, 20(7), 838847 (2015).

216. Li X, Patterson JT, Sarkar M et al. Site-Specific Dual Antibody Conjugation via Engineered Cysteine and Selenocysteine Residues. Bioconjug Chem, (2015).

217. Merten H, Brandl F, Pluckthun A, Zangemeister-Wittke U. Antibody-Drug Conjugates for Tumor Targeting-Novel Conjugation Chemistries and the Promise of non-lgG Binding Proteins. Bioconjug Chem, (2015). 